\title{
A Summary of Corrosion Properties of Al-Rich Solid Solution and Secondary Phase Particles in Al Alloys
}

\author{
Jichao Li and Jie Dang * \\ College of Materials Science and Engineering, Chongqing University, Chongqing 400044, China; \\ lijichaocn@gmail.com \\ * Correspondence: jiedang@cqu.edu.cn; Tel.: +86-23-6511-2631
}

Academic Editor: Hugo F. Lopez

Received: 28 November 2016; Accepted: 28 February 2017; Published: 7 March 2017

\begin{abstract}
The heterogeneous structure of $\mathrm{Al}$ alloys renders them susceptible to localized corrosion due to the different electrochemical properties existing in the Al-rich solid solution matrix and secondary phase particles. The galvanic interactions between these two phases can result in pit formation either through dissolution of the particles or corrosion of the matrix adjacent to the particles. This detrimentally localized corrosion behavior is closely related to the corrosion properties of the particles and the Al-rich matrix. The comprehensive characterization of this behavior under various and varying conditions is critical to understanding the mechanism of pit formation, selecting appropriate inhibitors, and developing protection strategies. The corrosion properties (corrosion potential, pitting potential and corrosion rate) of both secondary phase particles and Al-solid solutions in $\mathrm{Al}$ alloys are summarized in this review, aiming to provide a database for corrosion research applicable to the localized corrosion of $\mathrm{Al}$ alloys.
\end{abstract}

Keywords: Al alloys; Al-rich solid solution; secondary phase particles; corrosion potential; pitting potential; corrosion rate

\section{Introduction}

Alloying elements are added to aluminum to improve its mechanical properties such as the strength and toughness through precipitation strengthening and thermochemical processing $[1,2]$. The resulting microstructure in high-strength aluminum alloys is complex and includes both equilibrium and non-equilibrium phases [1,2]. Depending on the desired mechanical and corrosion properties, the total incorporated alloying elements can exceed $10 \%[1,2]$. Common alloying elements include $\mathrm{Cu}, \mathrm{Si}, \mathrm{Mg}, \mathrm{Mn}, \mathrm{Zn}$, and Li. Less common elements include deliberate additions including Ag, and $\mathrm{Zr}$, and impurities such as Fe [1,3,4].

Since alloying elements in the bulk alloy are typically present in concentrations far exceeding their equilibrium solubility under solid conditions, exposure to elevated temperatures during solidification and heat treatment leads to the formation of precipitates and large constituent, leading to a heterogeneous structure [1,4-6]. The consequence of the heterogeneous structure is the presence of a mixture of several phases in the alloy including Al-rich matrix phase and many solute-rich secondary phase particles [7]. The formation of this heterogeneous structure degrades the original corrosion resistance that would arise from the homogeneous structure in which the alloying elements are in the solid solution.

The compositions of secondary phase particles are complex. In fact, they may consist of several different phases, which sometimes cannot be accurately defined by a stoichiometric formula [8]. Commonly identified particles include, but are not limited to, $\mathrm{Mg}_{2} \mathrm{Si}, \mathrm{MgZn} \mathrm{n}_{2}, \mathrm{Al}_{20} \mathrm{Cu}_{2} \mathrm{Mn}_{3}, \mathrm{Al}_{12} \mathrm{Mn}_{3} \mathrm{Si}$, $\mathrm{Al}_{7} \mathrm{Cu}_{2} \mathrm{Fe}, \mathrm{Al}_{2} \mathrm{Cu}, \mathrm{Al}_{2} \mathrm{CuMg}, \mathrm{Al}_{3} \mathrm{Fe}, \mathrm{Al}_{12} \mathrm{Mg}_{2} \mathrm{Cr}, \mathrm{Al}_{20} \mathrm{Cu}_{2} \mathrm{Mn}_{3}, \mathrm{Al}_{6} \mathrm{Mn}, \mathrm{Al}_{3} \mathrm{Ti}, \mathrm{Al}_{3} \mathrm{Zr}, \mathrm{Al}_{3} \mathrm{Ta}, \mathrm{Mg}_{2} \mathrm{Al}_{3}$, $\mathrm{Al}_{32} \mathrm{Zn}_{49}, \mathrm{Al}_{2} \mathrm{CuLi}, \mathrm{Al}_{5} \mathrm{CuLi}$, and $\mathrm{Mg}_{2} \mathrm{Zn}_{11}[1,3-5,9,10]$. The size of these particles ranges from a few 
angstroms to a few micrometers, and they are present in different shapes including spheres, needles, lathes, plates, and more [11]. Buchheit et al. examined the secondary phase particles larger than 0.5 0.7 $\mu \mathrm{m}$ on AA2024-T3 and divided them into four categories [12]. The largest category included small and round $\mathrm{Al}_{2} \mathrm{CuMg}$, which was found to constitute about $66.2 \%$ of the particles by number and around $2.7 \%$ of the total surface area. A recent study by Boag et al. showed that approximately $40 \%$ of the total numbers of secondary phase particles are anodic relative to the surrounding matrix in AA 2024-T3 alloys, while the remaining $60 \%$ of particles are cathodic. The irregular "Al-Cu-Mn-Fe particles" comprised the second largest category and were defined as $\mathrm{Al}_{6}(\mathrm{Cu}, \mathrm{Fe}, \mathrm{Mn})$. The third largest category contained $\mathrm{Al}-\mathrm{Cu}-\mathrm{Fe}$ type particles $\left(\mathrm{Al}_{7} \mathrm{Cu}_{2} \mathrm{Fe}\right)$. The final category was identified as $\mathrm{Al}-\mathrm{Cu}-\mathrm{Mn}$, which is expressed as $(\mathrm{Al}, \mathrm{Cu})_{6} \mathrm{Mn}$. A detailed analysis using the advanced energy dispersive $\mathrm{X}$-ray spectroscopies reveals that the secondary phases usually mixed together, exhibiting multiphase particles and periphery phases around composite particles and clustering. The shape-classification may not be accurate criteria to judge the corrosion role. For example, some $\mathrm{Al}_{7} \mathrm{Cu}_{2} \mathrm{Fe}$ particles show similar shape to $\mathrm{Al}_{2} \mathrm{CuMg}$ and $\mathrm{Al}_{2} \mathrm{Cu}$, which are found to be indistinguishable in term of shape [8].

The formation mechanism of these particles varies and the induced effects in mechanical improvement of the alloy are also different [1,4,11]. Alloying elements such as $\mathrm{Cu}, \mathrm{Zn}, \mathrm{Mg}$, and $\mathrm{Li}$ generally have high solubility in $\mathrm{Al}$ at high temperature, and they form precipitates including $\mathrm{Al}_{2} \mathrm{Cu}, \mathrm{MgZn}_{2}, \mathrm{Mg}_{2} \mathrm{Al}_{3}, \mathrm{Al}_{2} \mathrm{Cu}, \mathrm{Al}_{2} \mathrm{CuLi}, \mathrm{Al}_{2} \mathrm{CuMg}, \mathrm{Al}_{6} \mathrm{CuLi}_{3}$ and $\mathrm{Al}_{32} \mathrm{Zn}_{49}$ during solidification or heat treatment. Impurities such as $\mathrm{Fe}$, which can have a high concentration depending on the impurity level of the alloy, can combine with $\mathrm{Cu}$ and $\mathrm{Al}$ to form large irregular constituents during solidification, with the size ranging from fractions of a micrometer to 20 micrometers [12]. These large constituent particles are expected to be harmful to the corrosion resistance of $\mathrm{Al}$ alloys not only due to their electrochemical properties but also their size and alloy composition fraction. Some minor alloying elements such as $\mathrm{Zr}$ and $\mathrm{Ti}$ combines with $\mathrm{Al}$ to form fine dispersoids such as $\mathrm{Al}_{3} \mathrm{Ti}, \mathrm{Al}_{3} \mathrm{Zr}, \mathrm{Al}_{3} \mathrm{Ta}$ [11]. These particles can refine grains and control the recrystallization. Their effect on the corrosion behavior of alloys is negligible since their concentration is very low and the particles are very fine [11].

Based on their electrochemical behavior, secondary phase particles can be divided into two groups: reactive particles with active elements (such as $\mathrm{Mg}, \mathrm{Li}$, and $\mathrm{Zn}$ ) and noble particles with noble elements (such as $\mathrm{Cu}$ and $\mathrm{Fe}$ ). When active alloying elements dominate a particle, they render the particle more active than the surrounding matrix, and these particles (or portions of these particles) subsequently dissolve as an anode. The induced localized corrosion results in deep pits [11]. For particles containing both active and noble elements, such as $\mathrm{Al}_{2} \mathrm{CuMg}$, the active alloying elements dissolve leaving behind remnants with noble elements such as $\mathrm{Cu}$. This is commonly referred to as dealloying corrosion as shown in Figure 1 [11-27]. Particles consisting primarily of noble elements generally possess a high corrosion potential, and act as a cathode to support the oxygen reduction reaction or hydrogen evolution. The adjacent matrix then dissolves at the matrix/particle interface, resulting in trenching morphology surrounding the particle as shown in Figure 2 [28-32]. However, the galvanic relations between the matrix and secondary phase particles may change with time and as environmental conditions are altered. For example, $\mathrm{Al}_{20} \mathrm{Cu}_{2} \mathrm{Mn}_{3}$ is a noble particle relative to the matrix, and its corrosion during exposure in $\mathrm{NaCl}$ solution at room temperature is minimal. However, it switches to an anode as temperature increases above $50{ }^{\circ} \mathrm{C}$, with $\mathrm{Al}$ selectively corroding, showing a dealloying pattern commonly observed on $\mathrm{Al}_{2} \mathrm{CuMg}$ [33]. 

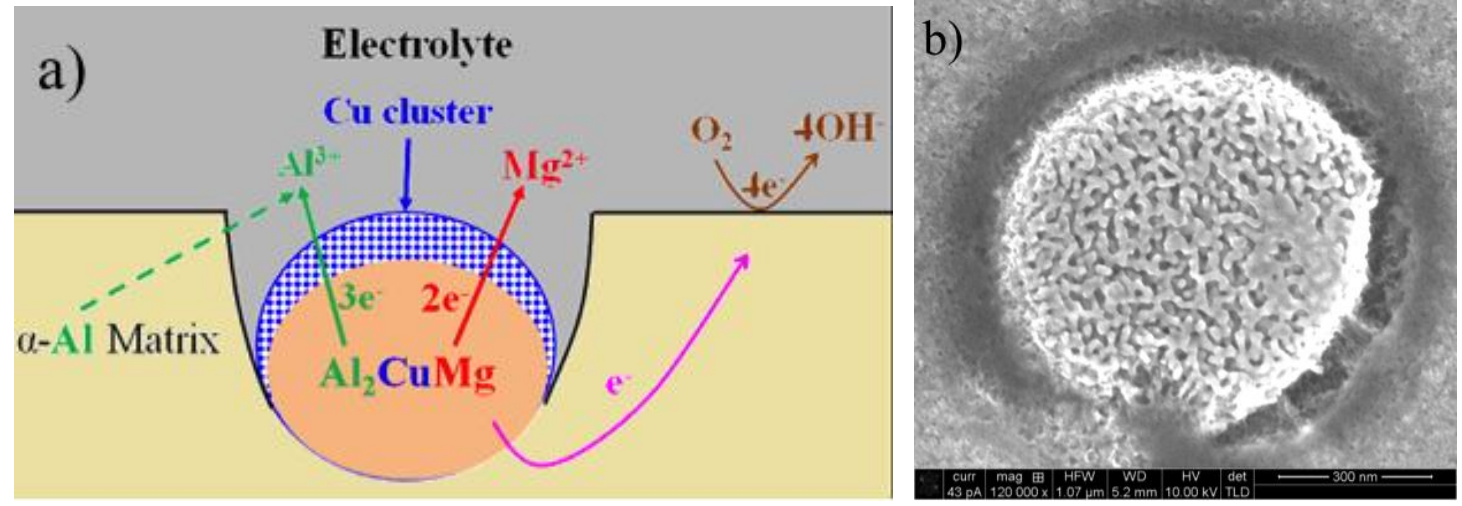

Figure 1. Schematic dealloying and subsequent trenching corrosion process of $\mathrm{Al}_{2} \mathrm{CuMg}$ in AA2024 (a); and scanning electron microscope (SEM) images of resulted corrosion morphology after 1-h exposure in aerated De-ionized water at $30^{\circ} \mathrm{C}(\mathbf{b})$.
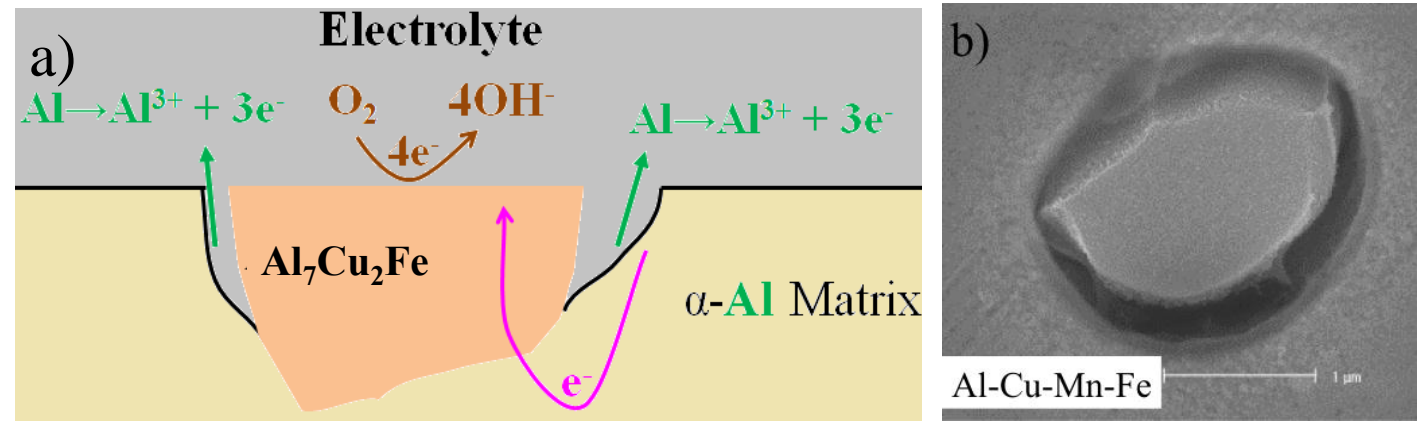

Figure 2. Schematic trenching corrosion process of $\mathrm{Al}_{7} \mathrm{Cu}_{2} \mathrm{Fe}$ in AA2024-T3 (a); and SEM image of the resulted corrosion morphology after 1 -h exposure in De-ionized water at $30^{\circ} \mathrm{C}(\mathbf{b})$.

Almost all localized corrosion in aluminum alloys is closely related to secondary phase particles, and their electrochemical properties are strongly dependent on environmental conditions such as temperature, $\mathrm{pH}$ and chloride ion $\left(\mathrm{Cl}^{-}\right)[11,17,33,34]$. To have a full understanding of localized corrosion, it is necessary to have comprehensive electrochemical characterization of secondary phase particles under a variety of conditions. This will provide a deeper understanding about localized corrosion and an aid in the development of strategies to inhibit localized corrosion. Furthermore, these basic corrosion data can be used to simulate pit initiation and propagation. One very successful method for determining the corrosion characteristics of secondary phase particles is the electrochemical microcell method, which uses a small capillary filled with electrolyte, the tip of which permits electrochemical analysis of specific particles [11,35-40]. This method has been employed with both synthesized intermetallic compounds (IMCs) and/or actual alloy substrates [11,17,34,41-47].

Using recent literature, this paper aims to collect and summarize the electrochemical properties of secondary phase particles commonly found in $\mathrm{Al}$ alloys and Al-rich solid solutions. The corrosion potential, pitting potential and corrosion rates of Al-rich solid solutions and secondary phase particles under various conditions are summarized in Table 1. 
Table 1. Corrosion potential, pitting potential and corrosion current density of Al-rich solid solution and second phase particles.

\begin{tabular}{|c|c|c|c|c|c|}
\hline Stoichiometry & $E_{\text {corr }}, \mathrm{V}_{\mathrm{SCE}}$ & $E_{\text {pit }}, \mathrm{V}_{\mathrm{SCE}}$ & $i_{\text {corr }}, \mu \mathrm{A} / \mathrm{cm}^{2}$ & Environment & Reference \\
\hline \multirow{8}{*}{ Al } & -0.679 & -0.545 & N/A & Aerated $0.01 \mathrm{M} \mathrm{NaCl}$ & [11] \\
\hline & -1.01 & -0.56 & 1.5 & $0.5 \mathrm{M} \mathrm{NaCl}, \mathrm{pH} 9.17$ & [48] \\
\hline & -0.823 & -0.610 & 3.9 & Aerated $0.1 \mathrm{M} \mathrm{NaCl}$ & [11] \\
\hline & -0.849 & -0.696 & $\mathrm{~N} / \mathrm{A}$ & Aerated $0.6 \mathrm{M} \mathrm{NaCl}$ & [11] \\
\hline & -0.823 & -0.568 & 0.87 & Aerated $0.1 \mathrm{M} \mathrm{NaCl}, 10^{\circ} \mathrm{C}$ & [49] \\
\hline & -0.942 & -0.637 & 1.09 & Aerated $0.1 \mathrm{M} \mathrm{NaCl}, 30^{\circ} \mathrm{C}$ & [49] \\
\hline & -0.99 & -0.67 & 1.73 & Aerated $0.1 \mathrm{M} \mathrm{NaCl}, 50^{\circ} \mathrm{C}$ & [49] \\
\hline & -1.22 & -0.673 & 1.65 & Aerated $0.1 \mathrm{M} \mathrm{NaCl}, 70{ }^{\circ} \mathrm{C}$ & [49] \\
\hline Al- $1 \% C u$, solid solution & -0.69 & N/A & N/A & Aerated 5 wt. $\% \mathrm{NaCl}$, pH 3.1-3.3 & [50] \\
\hline \multirow{3}{*}{$\mathrm{Al}-2 \% \mathrm{Cu}$} & -0.813 & -0.447 & N/A & Aerated $0.01 \mathrm{M} \mathrm{NaCl}$ & [11] \\
\hline & -0.672 & -0.471 & 1.3 & Aerated $0.1 \mathrm{M} \mathrm{NaCl}$ & [11] \\
\hline & -0.744 & -0.529 & N/A & Aerated $0.6 \mathrm{M} \mathrm{NaCl}$ & [11] \\
\hline \multirow{19}{*}{$\mathrm{Al}-4 \% \mathrm{Cu}$} & -0.856 & -0.389 & 0.94 & Aerated $0.1 \mathrm{M} \mathrm{NaCl}, 10^{\circ} \mathrm{C}$ & [33] \\
\hline & -0.894 & -0.380 & 0.94 & Aerated $0.1 \mathrm{M} \mathrm{NaCl}, 30^{\circ} \mathrm{C}$ & [33] \\
\hline & -0.813 & -0.400 & 1.08 & Aerated $0.1 \mathrm{M} \mathrm{NaCl}, 50^{\circ} \mathrm{C}$ & [33] \\
\hline & -0.784 & -0.352 & 2.97 & Aerated $0.1 \mathrm{M} \mathrm{NaCl}, 70^{\circ} \mathrm{C}$ & [33] \\
\hline & -0.985 & -0.458 & 0.31 & $0.1 \mathrm{M} \mathrm{NaCl}+4 \mathrm{mM} \mathrm{CeCl}_{3}, 30^{\circ} \mathrm{C}$ & [51] \\
\hline & -0.950 & -0.396 & 0.32 & $0.1 \mathrm{M} \mathrm{NaCl}+4 \mathrm{mM} \mathrm{LaCl}_{3}, 30^{\circ} \mathrm{C}$ & [42] \\
\hline & -0.91 & -0.45 & 0.22 & $0.1 \mathrm{M} \mathrm{NaCl}+4 \mathrm{mM} \mathrm{PrCl}_{3}, 30^{\circ} \mathrm{C}$ & [42] \\
\hline & -0.877 & -0.21 & 0.11 & $0.1 \mathrm{M} \mathrm{NaCl}+1 \mathrm{mM} \mathrm{K}_{2} \mathrm{CrO}_{4}, 30^{\circ} \mathrm{C}$ & [42] \\
\hline & -1.05 & -0.307 & 0.46 & $0.1 \mathrm{M} \mathrm{NaCl}+4 \mathrm{mM} \mathrm{CeCl}_{3}, 50^{\circ} \mathrm{C}$ & [51] \\
\hline & -1.05 & -0.397 & 1.6 & $0.1 \mathrm{M} \mathrm{NaCl}+4 \mathrm{mM} \mathrm{CeCl}_{3}, 70^{\circ} \mathrm{C}$ & [51] \\
\hline & $-0.583 \sim-0.589$ & N/A & N/A & De-aerated $1 \mathrm{M} \mathrm{NaCl}+1$ wt. $\% \mathrm{H}_{2} \mathrm{O}_{2}+0.1 \%$ proof stress & [52] \\
\hline & $-0.762 \sim-0.606$ & N/A & N/A & $\begin{array}{l}0.02 \mathrm{~d} \text { to } 10 \text { day aging at } 1700^{\circ} \mathrm{C} \text {, de-aerated } 1 \mathrm{M} \mathrm{NaCl}+ \\
1 \mathrm{wt} . \% \mathrm{H}_{2} \mathrm{O}_{2}+0.1 \% \text { proof stress }\end{array}$ & [52] \\
\hline & $-1.18^{\mathrm{a}}$ & -0.6 & N/A & $0.1 \mathrm{M} \mathrm{NaCl}, \mathrm{pH} 10$ & [52] \\
\hline & $-1.1^{\mathrm{a}}$ & $-0.75 \sim-0.69$ & N/A & $0.01 \mathrm{~d}$ to 10 day aging at $170^{\circ} \mathrm{C}, 0.1 \mathrm{M} \mathrm{NaCl}, \mathrm{pH} 10$ & [52] \\
\hline & -0.87 & -0.47 & 2.9 & $0.5 \mathrm{M} \mathrm{NaCl}, \mathrm{pH} 9.17$ & [48] \\
\hline & -1.18 & -0.38 & 0.54 & $0.5 \mathrm{M} \mathrm{NaCl}+10 \mathrm{mM} \mathrm{NaVO}_{3}, \mathrm{pH} 9.17$ & [48] \\
\hline & -0.750 & -0.418 & N/A & Aerated $0.01 \mathrm{M} \mathrm{NaCl}$ & [11] \\
\hline & -0.602 & -0.406 & 2.3 & Aerated $0.1 \mathrm{M} \mathrm{NaCl}$ & [11] \\
\hline & -0.642 & -0.465 & $\mathrm{~N} / \mathrm{A}$ & Aerated $0.6 \mathrm{M} \mathrm{NaCl}$ & [11] \\
\hline $\mathrm{Al}-x \mathrm{Cu}(x=0.011 \sim 8.15$ wt. $\%)$ & $\mathrm{N} / \mathrm{A}$ & $-0.76 \sim-0.649^{\text {a }}$ & N/A & $1 \mathrm{M} \mathrm{NaCl}, \mathrm{pH} 10$ & [53] \\
\hline $\mathrm{Al}-3 \% \mathrm{Mg}$ & -0.78 & N/A & N/A & Solid solution, aerated 5 wt. \% $\mathrm{NaCl} \mathrm{pH}$ 3.1-3.3 & [50] \\
\hline $\operatorname{Al}-x \operatorname{Mg}(x=0.01 \sim 4.51$ wt. $\%)$ & N/A & $-0.758 \sim-0.82^{a}$ & N/A & $1 \mathrm{M} \mathrm{NaCl}, \mathrm{pH} 10$ & [53] \\
\hline $\mathrm{Al}-1 \% \mathrm{Mn}$ & -0.73 & N/A & N/A & Solid solution, aerated 5 wt. \% NaCl pH 3.1-3.3 & [50] \\
\hline $\operatorname{Al}-x \operatorname{Mn}(x=0 \sim 2$ wt. $\%)$ & $-0.944 \sim-0.812^{\mathrm{a}}$ & N/A & N/A & Solid solution, in $53 \mathrm{~g} / \mathrm{L} \mathrm{NaCl}+3 \mathrm{~g} / \mathrm{L} \mathrm{H}_{2} \mathrm{O}_{2}$ & [54] \\
\hline $\operatorname{Al}-x \operatorname{Mn}(x=0 \sim 2$ wt. $\%)$ & $-1.01 \sim-1.14^{\mathrm{a}}$ & $-0.70 \sim-0.76$ & N/A & De-aerated $\mathrm{AlCl}_{3}, \mathrm{pH} 2.5$ & [55] \\
\hline $\mathrm{Al}-x \operatorname{Mn}(x=0.011 \sim 4.94$ wt. $\%)$ & N/A & $-0.76 \sim-0.718^{\mathrm{a}}$ & N/A & $1 \mathrm{M} \mathrm{NaCl}, \mathrm{pH} 10$ & [53] \\
\hline Al-0.1 Li, solid solution & -1.067 & -0.727 & $\mathrm{~N} / \mathrm{A}$ & Deaerated agitated $3.5 \% \mathrm{NaCl}$ & [56] \\
\hline Al-2.85 $\mathrm{Li}\left(0.25 \sim 24 \mathrm{~h}\right.$ aging at $\left.200^{\circ} \mathrm{C}\right)$ & $-1.13 \sim-1.146$ & $-0.692 \sim-0.725$ & N/A & Deaerated agitated $3.5 \% \mathrm{NaCl}$ & [56] \\
\hline
\end{tabular}


Table 1. Cont

\begin{tabular}{|c|c|c|c|c|c|}
\hline Stoichiometry & $E_{\text {corr }}, \mathbf{V}_{\mathrm{SCE}}$ & $E_{\mathrm{pit}}, \mathrm{V}_{\mathrm{SCE}}$ & $i_{\text {corr }} \mu \mathrm{\mu} / \mathrm{cm}^{2}$ & Environment & Reference \\
\hline $\begin{array}{c}\text { Al-2.78Li- } 0.32 \mathrm{Mn}, \\
\left(0.25 \sim 336 \mathrm{~h} \text { aging at } 200{ }^{\circ} \mathrm{C}\right) \\
\end{array}$ & $-1.193 \sim-1.36$ & $-0.693 \sim-0.721$ & $\mathrm{~N} / \mathrm{A}$ & Deaerated agitated $3.5 \% \mathrm{NaCl}$ & [56] \\
\hline $\begin{array}{c}\text { Al-2.76Li- } 0.14 \mathrm{Zr} \\
\left(0.25 \sim 336 \mathrm{~h} \text { aging at } 200{ }^{\circ} \mathrm{C}\right)\end{array}$ & $-1.61 \sim-1.41$ & $-0.765 \sim-0.705$ & N/A & Deaerated agitated $3.5 \% \mathrm{NaCl}$ & [56] \\
\hline $\mathrm{Al}-0.04 \mathrm{Fe}$ & -0.76 & $\mathrm{~N} / \mathrm{A}$ & N/A & Solid solution, aerated s $5 \mathrm{wt}$ \% $\mathrm{NaCl} \mathrm{pH} \mathrm{3.1-3.3}$ & [50] \\
\hline $\mathrm{Al}-x \mathrm{Fe}(x=0.013 \sim 3.38$ wt. $\%)$ & $\mathrm{N} / \mathrm{A}$ & $-0.77 \sim-0.758^{a}$ & $\mathrm{~N} / \mathrm{A}$ & $1 \mathrm{M} \mathrm{NaCl}, \mathrm{pH} 10$ & {$[53]$} \\
\hline $\mathrm{Al}-x \mathrm{Si}(x=0.05 \sim 1.66$ wt. $\%)$ & N/A & $-0.75 \sim-0.7^{a}$ & $\mathrm{~N} / \mathrm{A}$ & $1 \mathrm{M} \mathrm{NaCl}, \mathrm{pH} 10$ & [53] \\
\hline \multirow{3}{*}{$\mathrm{Al}_{2} \mathrm{Cu}(\theta)$} & $\begin{array}{l}-0.592 \\
-0.665 \\
-0.695 \\
-0.53 \\
-0.57 \\
-0.557 \\
-0.578 \\
-0.53 \\
-0.55 \\
-0.52 \\
-0.45\end{array}$ & $\begin{array}{l}-0.434 \\
-0.544 \\
-0.652 \\
-0.42 \\
-0.53 \\
-0.467 \\
-0.495 \\
-0.5 \\
-0.5 \\
-0.33 \\
\text { No }\end{array}$ & $\begin{array}{c}\text { N/A } \\
7.3 \\
\text { N/A } \\
4.9 \\
11 \\
0.9 \\
1.6 \\
13 \\
13 \\
0.13 \\
1.82 \\
\end{array}$ & $\begin{array}{c}\text { Aerated } 0.01 \mathrm{M} \mathrm{NaCl} \\
\text { Aerated } 0.1 \mathrm{M} \mathrm{NaCl} \\
\text { Aerated } 0.6 \mathrm{M} \mathrm{NaCl} \\
\text { Aerated } 0.1 \mathrm{M} \mathrm{NaCl}, 30^{\circ} \mathrm{C} \\
0.1 \mathrm{M} \mathrm{NaCl}+4 \mathrm{mM} \mathrm{CeCl}_{3}, 30^{\circ} \mathrm{C} \\
0.1 \mathrm{M} \mathrm{NaCl}+4 \mathrm{mM} \mathrm{CeCl}_{3}, 50^{\circ} \mathrm{C} \\
0.1 \mathrm{M} \mathrm{NaCl}+4 \mathrm{mM} \mathrm{CeCl}_{3}, 70^{\circ} \mathrm{C} \\
0.1 \mathrm{M} \mathrm{NaCl}+4 \mathrm{mM} \mathrm{PrCl}_{3}, 30^{\circ} \mathrm{C} \\
0.1 \mathrm{M} \mathrm{NaCl}+4 \mathrm{mM} \mathrm{LaCl}_{3}, 30^{\circ} \mathrm{C} \\
0.1 \mathrm{M} \mathrm{NaCl}+1 \mathrm{mM} \mathrm{K}_{2} \mathrm{CrO}_{4}, 30^{\circ} \mathrm{C} \\
0.1 \mathrm{M} \mathrm{NaCl}, 10^{\circ} \mathrm{C} \\
\end{array}$ & $\begin{array}{c}{[11]} \\
{[11]} \\
{[11]} \\
{[33,42]} \\
{[42,51]} \\
{[51]} \\
{[51]} \\
{[42]} \\
{[42]} \\
{[42]} \\
{[33]} \\
\end{array}$ \\
\hline & $\begin{array}{l}-0.57 \\
-0.56 \\
-0.546 \\
-0.665 \\
-0.739 \\
-0.743 \\
-0.46^{\mathrm{a}} \\
-0.5^{\mathrm{a}} \\
-0.68^{\mathrm{a}} \\
-0.93^{\mathrm{a}} \\
-1.16^{\mathrm{a}}\end{array}$ & $\begin{array}{l}-0.43 \\
-0.487 \\
-0.458 \\
-0.544 \\
-0.408 \\
-0.407 \\
\text { N/A } \\
\text { N/A } \\
\text { N/A } \\
\text { N/A } \\
\text { N/A }\end{array}$ & $\begin{array}{l}8.35 \\
27.4 \\
-38^{\mathrm{c}} \\
-41^{\mathrm{c}} \\
-110^{\mathrm{c}} \\
-38^{\mathrm{c}} \\
\text { N/A } \\
\text { N/A } \\
\text { N/A } \\
\text { N/A } \\
\text { N/A }\end{array}$ & $\begin{array}{c}0.1 \mathrm{M} \mathrm{NaCl}, 50^{\circ} \mathrm{C} \\
0.1 \mathrm{M} \mathrm{NaCl}, 70^{\circ} \mathrm{C} \\
0.1 \mathrm{M} \mathrm{NaCl}, \mathrm{pH} 2.5 \\
0.1 \mathrm{M} \mathrm{NaCl}, \mathrm{pH} 6 \\
0.1 \mathrm{M} \mathrm{NaCl}, \mathrm{pH} 10 \\
0.1 \mathrm{M} \mathrm{NaCl}, \mathrm{pH} 12.5 \\
\text { Deaerated } 0.5 \mathrm{M} \mathrm{H}_{2} \mathrm{SO}_{4}, \mathrm{pH} 0.4 \\
\text { Deaerated } 0.5 \mathrm{M} \mathrm{NaSO}_{4}+0.005 \mathrm{H}_{2} \mathrm{SO}_{4}, \mathrm{pH} 2.8 \\
\text { Deaerated } 0.5 \mathrm{M} \mathrm{Na}_{2} \mathrm{SO}_{4}, \mathrm{pH} 7.7 \\
\text { Deaerated } 0.5 \mathrm{M} \mathrm{Na}_{2} \mathrm{SO}_{4}+0.01 \mathrm{NaOH}, \mathrm{pH} 10.4 \\
\text { Deaerated } 1 \mathrm{M} \mathrm{NaOH}, \mathrm{pH} 13.8 \\
\end{array}$ & $\begin{array}{l}{[33]} \\
{[33]} \\
{[34]} \\
{[34]} \\
{[34]} \\
{[34]} \\
{[57]} \\
{[57]} \\
{[57]} \\
{[57]} \\
{[57]}\end{array}$ \\
\hline & $\begin{array}{l}-0.625 \\
-0.7^{\mathrm{a}} \\
-0.65^{\mathrm{a}} \\
-0.59 \\
-0.91 \\
-0.405^{\mathrm{a}} \\
-0.436^{\mathrm{a}} \\
-0.374^{\mathrm{a}} \\
\end{array}$ & $\begin{array}{l}\text { No } \\
\text { N/A } \\
\text { N/A } \\
-0.44 \\
-0.34 \\
\text { N/A } \\
\text { N/A } \\
\text { N/A } \\
\end{array}$ & $\begin{array}{l}\text { N/A } \\
\text { N/A } \\
\text { N/A } \\
13 \\
4.5 \\
\text { N/A } \\
\text { N/A } \\
\text { N/A }\end{array}$ & $\begin{array}{c}\text { De-aerated } 1 \mathrm{M} \mathrm{NaCl} \\
\text { Aerated } 0.6 \mathrm{M} \mathrm{NaCl} \\
0.51 \mathrm{M} \mathrm{NaCl}, \mathrm{pH} 1.2 \\
0.5 \mathrm{M} \mathrm{NaCl} \text { pH } 9.17 \\
0.5 \mathrm{M} \mathrm{NaCl}^{2}+10 \mathrm{mM} \mathrm{NaVO} 3, \mathrm{pH} 9.17 \\
0.1 \mathrm{M} \mathrm{Na}_{2} \mathrm{SO}_{4}+0.005 \mathrm{M} \mathrm{NaCl}, \mathrm{pH} 6 \\
0.1 \mathrm{M} \mathrm{Na}_{2} \mathrm{SO}_{4}+0.005 \mathrm{M} \mathrm{NaCl} \mathrm{pH} 3 \\
0.1 \mathrm{M} \mathrm{Na}_{2} \mathrm{SO}_{4}+0.005 \mathrm{M} \mathrm{NaCl}, \mathrm{pH} 10 \\
\end{array}$ & $\begin{array}{l}{[52]} \\
{[58]} \\
{[58]} \\
{[48]} \\
{[48]} \\
{[31]} \\
{[31]} \\
{[31]} \\
\end{array}$ \\
\hline $\mathrm{Al}_{3} \mathrm{Ta}$ & $\begin{array}{c}-0.346 \sim-0.386 \\
-0.82 \\
-1.251\end{array}$ & $\begin{array}{l}\text { N/A } \\
\text { N/A } \\
\text { N/A }\end{array}$ & $\begin{array}{l}\text { N/A } \\
\text { N/A } \\
\text { N/A }\end{array}$ & $\begin{array}{c}0.1 \mathrm{M} \mathrm{C}_{4} \mathrm{H}_{4} \mathrm{O}_{6}(\mathrm{pH} 2), 0.1 \mathrm{M} \mathrm{H}_{3} \mathrm{BO}_{4}(\mathrm{pH} 4.8) \\
0.1 \mathrm{M} \mathrm{H}_{3} \mathrm{BO}_{4}+0.1 \mathrm{M} \mathrm{Na}_{2} \mathrm{~B}_{4} \mathrm{O}_{7}(\mathrm{pH} \mathrm{6.9)} \\
0.1 \mathrm{Ma} \mathrm{a}_{2} \mathrm{~B}_{4} \mathrm{O}_{7}(\mathrm{pH} 9.3) \\
0.1 \mathrm{M} \mathrm{Na}_{2} \mathrm{~B}_{4} \mathrm{O}_{7}+0.1 \mathrm{M} \mathrm{NaOH}(\mathrm{pH} 12)\end{array}$ & $\begin{array}{l}{[59]} \\
{[59]} \\
{[59]}\end{array}$ \\
\hline
\end{tabular}


Table 1. Cont

\begin{tabular}{|c|c|c|c|c|c|}
\hline Stoichiometry & $E_{\text {corr }}, \mathrm{V}_{\mathrm{SCE}}$ & $E_{\mathrm{pit}}, \mathrm{V}_{\mathrm{SCE}}$ & 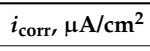 & Environment & Reference \\
\hline $\mathrm{Al}_{3} \mathrm{Zr}$ & $\begin{array}{l}-0.752 \\
-0.776 \\
-0.801\end{array}$ & $\begin{array}{l}-0.223 \\
-0.275 \\
-0.346\end{array}$ & $\begin{array}{c}\text { N/A } \\
2.5 \\
\text { N/A }\end{array}$ & $\begin{array}{l}\text { Aerated } 0.01 \mathrm{M} \mathrm{NaCl} \\
\text { Aerated } 0.1 \mathrm{M} \mathrm{NaCl} \\
\text { Aerated } 0.6 \mathrm{M} \mathrm{NaCl}\end{array}$ & $\begin{array}{l}{[11]} \\
{[11]} \\
{[11]}\end{array}$ \\
\hline $\mathrm{Al}_{3} \mathrm{Ti}$ & $\begin{array}{l}-0.609 \\
-0.620 \\
-0.603 \\
-0.799\end{array}$ & $\begin{array}{l}-0.139 \\
-0.232 \\
-0.225 \\
-0.646\end{array}$ & $\begin{array}{l}\text { N/A } \\
\text { N/A } \\
0.56 \\
\text { N/A }\end{array}$ & $\begin{array}{c}0.1 \mathrm{M} \mathrm{NaCl}+1 \mathrm{mM} \mathrm{K}_{2} \mathrm{CrO}_{4}, 30^{\circ} \mathrm{C} \\
\text { Aerated } 0.01 \mathrm{M} \mathrm{NaCl} \\
\text { Aerated } 0.1 \mathrm{M} \mathrm{NaCl} \\
\text { Aerated } 0.6 \mathrm{M} \mathrm{NaCl}\end{array}$ & $\begin{array}{l}{[42]} \\
{[11]} \\
{[11]} \\
{[11]}\end{array}$ \\
\hline \multirow[b]{2}{*}{$\mathrm{Mg}_{2} \mathrm{Si}$} & $\begin{array}{l}-1.16 \\
-1.15^{\mathrm{a}} \\
-1.4^{\mathrm{a}} \\
-1.03^{\mathrm{a}} \\
-1.18^{\mathrm{a}} \\
-1.23^{\mathrm{a}}\end{array}$ & $\begin{array}{c}\text { N/A } \\
\text { N/A } \\
\text { N/A } \\
-0.273^{\text {a }} \\
\text { No } \\
\text { No }\end{array}$ & $\begin{array}{l}1.28 \\
\text { N/A } \\
\text { N/A } \\
\text { N/A } \\
\text { N/A } \\
\text { N/A }\end{array}$ & $\begin{array}{c}\text { 3.5\% NaCl solution } \\
\text { Aerated } 0.6 \mathrm{M} \mathrm{NaCl} \\
0.51 \mathrm{M} \mathrm{NaCl}, \mathrm{pH} 1.2 \\
0.01 \mathrm{M} \mathrm{NaCl}, \mathrm{pH} 13 \\
0.01 \mathrm{M} \mathrm{NaCl}, \mathrm{pH} 6 \\
0.01 \mathrm{M} \mathrm{NaCl}, \mathrm{pH} 2\end{array}$ & $\begin{array}{l}{[60]} \\
{[58]} \\
{[58]} \\
{[61]} \\
{[61]} \\
{[61]}\end{array}$ \\
\hline & $\begin{array}{c}-1.355 \\
-1.538 \\
-1.536 \\
-1.408 \\
-1.538 \\
-1.43 \\
-1.553\end{array}$ & $\begin{array}{l}\text { No } \\
\text { No } \\
\text { No } \\
\text { No } \\
\text { No } \\
\text { No } \\
-0.951\end{array}$ & $\begin{array}{c}\mathrm{N} / \mathrm{A} \\
7.7 \\
\mathrm{~N} / \mathrm{A} \\
3400^{\mathrm{c}} \\
340^{\mathrm{c}} \\
960^{\mathrm{c}} \\
50^{\mathrm{c}}\end{array}$ & $\begin{array}{c}\text { Aerated } 0.01 \mathrm{M} \mathrm{NaCl} \\
\text { Aerated } 0.1 \mathrm{M} \mathrm{NaCl} \\
\text { Aerated } 0.6 \mathrm{M} \mathrm{NaCl} \\
0.1 \mathrm{M} \mathrm{NaCl}, \mathrm{pH} 2.5 \\
0.1 \mathrm{M} \mathrm{NaCl}, \mathrm{pH} 6 \\
0.1 \mathrm{M} \mathrm{NaCl} \text { pH } 10 \\
0.1 \mathrm{M} \mathrm{NaCl}, \mathrm{pH} 12.5\end{array}$ & $\begin{array}{l}{[11]} \\
{[11]} \\
{[11]} \\
{[34]} \\
{[34]} \\
{[34]} \\
{[34]}\end{array}$ \\
\hline $\begin{array}{c}\mathrm{Al}_{2} \mathrm{CuLi} \\
\text { (T1 phase) }\end{array}$ & $\begin{array}{l}-1.096 \\
-1.094 \\
-0.803 \\
-1.076\end{array}$ & $\begin{array}{l}-0.723 \\
-0.756 \\
\mathrm{~N} / \mathrm{A} \\
-0.73\end{array}$ & $\begin{array}{c}140 \sim 6.8^{\mathrm{a}, \mathrm{b}} \\
\mathrm{N} / \mathrm{A} \\
\mathrm{N} / \mathrm{A} \\
102\end{array}$ & $\begin{array}{c}\text { Aerated } 0.6 \mathrm{M} \mathrm{NaCl} \\
\text { De-aerated } 0.6 \mathrm{M} \mathrm{NaCl} \\
\text { ASTM G69 } \\
3.5 \text { wt. } \% \mathrm{NaCl}\end{array}$ & $\begin{array}{l}{[62]} \\
{[62]} \\
{[62]} \\
{[63]}\end{array}$ \\
\hline $\mathrm{Al}_{5(6)} \mathrm{CuLi}_{3}$ & -1.228 & -0.76 & 114 & 3.5 wt. $\% \mathrm{NaCl}$ & [63] \\
\hline $\mathrm{Al}_{32} \mathrm{Zn}_{49}$ & $\begin{array}{l}-1.009 \\
-1.004 \\
-1.063 \\
\end{array}$ & $\begin{array}{l}\text { No } \\
\text { No } \\
\text { No }\end{array}$ & $\begin{array}{c}\mathrm{N} / \mathrm{A} \\
14 \\
\mathrm{~N} / \mathrm{A} \\
\end{array}$ & $\begin{array}{l}\text { Aerated } 0.01 \mathrm{M} \mathrm{NaCl} \\
\text { Aerated } 0.1 \mathrm{M} \mathrm{NaCl} \\
\text { Aerated } 0.6 \mathrm{M} \mathrm{NaCl} \\
\end{array}$ & $\begin{array}{l}{[11]} \\
{[11]} \\
{[11]}\end{array}$ \\
\hline \multirow[t]{2}{*}{$\mathrm{Al}_{2} \mathrm{CuMg}$ (S phase) } & $\begin{array}{l}-0.95^{\mathrm{a}} \\
-0.95^{\mathrm{a}} \\
-0.92 \\
-1.06 \\
-0.956 \\
-0.883 \\
-1.061 \\
-0.93 \\
-0.93 \\
-0.750\end{array}$ & $\begin{array}{c}\text { N/A } \\
\text { N/A } \\
-0.29 \\
-0.60 \\
0.108 \\
0.080 \\
0.135 \\
\text { N/A } \\
\text { N/A } \\
\text { No }\end{array}$ & $\begin{array}{c}\text { N/A } \\
\text { N/A } \\
22 \\
2.8 \\
\text { N/A } \\
2 \\
\text { N/A } \\
\text { N/A } \\
\text { N/A } \\
-20^{c}\end{array}$ & $\begin{array}{c}\text { Aerated } 0.6 \mathrm{M} \mathrm{NaCl} \\
0.51 \mathrm{M} \mathrm{NaCl}, \mathrm{pH} 1.2 \\
0.5 \mathrm{M} \mathrm{NaCl}, \mathrm{pH} 9.17 \\
0.5 \mathrm{M} \mathrm{NaCl}+10 \mathrm{mM} \mathrm{NaVO}_{3}, \mathrm{pH} 9.17 \\
\text { Aerated } 0.01 \mathrm{M} \mathrm{NaCl} \\
\text { Aerated } 0.1 \mathrm{M} \mathrm{NaCl} \\
\text { Aerated } 0.6 \mathrm{M} \mathrm{NaCl} \\
\text { Aerated } 0.5 \mathrm{M} \mathrm{NaCl} \\
\text { De-aerated } 0.5 \mathrm{M} \mathrm{NaCl} \\
0.1 \mathrm{M} \mathrm{NaCl}, \mathrm{pH} 2.5\end{array}$ & $\begin{array}{c}{[58]} \\
{[58]} \\
{[48]} \\
{[48]} \\
{[11,64]} \\
{[11]} \\
{[11]} \\
{[21]} \\
{[21]} \\
{[34]}\end{array}$ \\
\hline & $\begin{array}{l}-0.883 \\
-0.85 \\
-0.67 \\
-0.86^{a} \\
-0.87^{a} \\
-0.873^{a}\end{array}$ & $\begin{array}{l}0.08 \\
-0.39 \\
-0.35 \\
\text { N/A } \\
\text { N/A } \\
\text { N/A }\end{array}$ & $\begin{array}{l} \pm 10^{\mathrm{c}} \\
\pm 1^{\mathrm{c}} \\
-36^{\mathrm{c}} \\
\text { N/A } \\
\text { N/A } \\
\text { N/A }\end{array}$ & $\begin{array}{c}0.1 \mathrm{M} \mathrm{NaCl}, \mathrm{pH} 6 \\
0.1 \mathrm{M} \mathrm{NaCl}, \mathrm{pH} 10 \\
0.1 \mathrm{M} \mathrm{NaCl}^{\mathrm{pH}} 12.5 \\
0.1 \mathrm{M} \mathrm{Na}_{2} \mathrm{SO}_{4}+0.005 \mathrm{M} \mathrm{NaCl}, \mathrm{pH} 6 \\
0.1 \mathrm{M} \mathrm{Na}_{2} \mathrm{SO}_{4}+0.005 \mathrm{M} \mathrm{NaCl}, \mathrm{pH} 3 \\
0.1 \mathrm{M} \mathrm{Na}_{2} \mathrm{SO}_{4}+0.005 \mathrm{M} \mathrm{NaCl}, \mathrm{pH} 10\end{array}$ & $\begin{array}{l}{[34]} \\
{[34]} \\
{[34]} \\
{[31]} \\
{[31]} \\
{[31]}\end{array}$ \\
\hline
\end{tabular}


Table 1. Cont

\begin{tabular}{|c|c|c|c|c|c|}
\hline Stoichiometry & $E_{\text {corr }}, \mathbf{V}_{\mathrm{SCE}}$ & $E_{\text {pit }}, V_{\mathrm{SCE}}$ & $i_{\text {corr }} \mu \mathrm{A} / \mathrm{cm}^{2}$ & Environment & Reference \\
\hline $\begin{array}{c}\mathrm{Mg}_{2} \mathrm{Al}_{3} \\
\text { ( } \beta \text { phase) }\end{array}$ & $\begin{array}{l}-1.225 \\
-1.197 \\
-1.313 \\
-1.31 \\
-1.435 \\
-1.124 \\
-1.013 \\
-1.162\end{array}$ & $\begin{array}{l}\text { No } \\
-0.88^{\mathrm{a}} \\
-0.9^{\mathrm{a}} \\
-0.85^{\mathrm{a}} \\
-0.9^{\mathrm{a}} \\
-0.818 \\
-0.846 \\
-0.959\end{array}$ & $\begin{array}{c}102 \\
2.3 \\
2.4 \\
1.2 \\
9.6 \\
\text { N/A } \\
4.8 \\
\text { N/A }\end{array}$ & $\begin{array}{c}0.01 \mathrm{M} \mathrm{NaCl}, \mathrm{pH} 2 \\
0.01 \mathrm{M} \mathrm{NaCl} \text {, } 4 \mathrm{4} 4 \\
0.01 \mathrm{M} \mathrm{NaCl} \mathrm{pH} 6 \\
0.01 \mathrm{M} \mathrm{NaCl}, \mathrm{pH} 8 \\
0.01 \mathrm{M} \mathrm{NaCl}, \mathrm{pH} 10 \\
\text { Aerated } 0.01 \mathrm{M} \mathrm{NaCl} \\
\text { Aerated } 0.1 \mathrm{M} \mathrm{NaCl} \\
\text { Aerated } 0.6 \mathrm{M} \mathrm{NaCl}\end{array}$ & $\begin{array}{l}{[65]} \\
{[65]} \\
{[65]} \\
{[65]} \\
{[65]} \\
{[11]} \\
{[11]} \\
{[11]}\end{array}$ \\
\hline $\mathrm{Mg}_{2} \mathrm{Al}_{3}$ (powder) & $\begin{array}{l}-1.43 \\
-1.39 \\
\end{array}$ & $\begin{array}{l}\text { N/A } \\
\text { N/A }\end{array}$ & $\begin{array}{l}\text { N/A } \\
\text { N/A }\end{array}$ & $\begin{array}{l}0.6 \mathrm{M} \mathrm{NaCl} \\
0.6 \mathrm{M} \mathrm{KOH}\end{array}$ & $\begin{array}{l}{[66]} \\
{[66]}\end{array}$ \\
\hline $\mathrm{Al}_{6} \mathrm{Mn}_{0.6} \mathrm{Fe}_{0.3}$ & $-0.7^{\mathrm{a}}$ & $\mathrm{N} / \mathrm{A}$ & $\mathrm{N} / \mathrm{A}$ & De-aerated $\mathrm{AlCl}_{3}, \mathrm{pH} 2.5$ & [55] \\
\hline $\mathrm{Al}_{6} \mathrm{Mn}$ & $\begin{array}{l}-1.059^{\mathrm{a}} \\
-0.839 \\
-0.779 \\
-0.913\end{array}$ & $\begin{array}{l}\mathrm{N} / \mathrm{A} \\
-0.485 \\
-0.755 \\
-0.778 \\
\end{array}$ & $\begin{array}{c}-0.69^{\mathrm{a}} \\
\text { N/A } \\
6.3 \\
\text { N/A }\end{array}$ & $\begin{array}{c}\text { De-aerated } \mathrm{AlCl}_{3}, \mathrm{pH} 2.5 \\
\text { Aerated } 0.01 \mathrm{M} \mathrm{NaCl} \\
\text { Aerated } 0.1 \mathrm{M} \mathrm{NaCl} \\
\text { Aerated } 0.6 \mathrm{M} \mathrm{NaCl}\end{array}$ & $\begin{array}{l}55] \\
{[11]} \\
{[11]} \\
{[11]}\end{array}$ \\
\hline $\mathrm{AlFe}_{3}$ & $\begin{array}{l}-0.605 \\
-0.550\end{array}$ & $\begin{array}{l}0.11 \\
0.11\end{array}$ & $\begin{array}{l}\text { N/A } \\
\text { N/A }\end{array}$ & $\begin{array}{l}0 \mathrm{M} \mathrm{KCl} \text { in aerated boric-borate solution ( } \mathrm{pH} 8.4) \\
0.1 \mathrm{M} \mathrm{KCl} \text { in aerated boric-borate solution ( } \mathrm{pH} 8.4)\end{array}$ & $\begin{array}{l}{[67]} \\
{[67]}\end{array}$ \\
\hline $\mathrm{AlFe}_{3} 0.2 \mathrm{Si}$ & $\begin{array}{l}-0.64 \\
-0.585 \\
\end{array}$ & $\begin{array}{l}0.215 \\
0.215\end{array}$ & $\begin{array}{l}\text { N/A } \\
\text { N/A }\end{array}$ & $\begin{array}{l}0 \mathrm{M} \mathrm{KCl} \text { in aerated boric-borate solution }(\mathrm{pH} 8.4) \\
0.1 \mathrm{M} \mathrm{KCl} \text { in aerated boric-borate solution }(\mathrm{pH} 8.4)\end{array}$ & $\begin{array}{l}{[67]} \\
{[67]} \\
\end{array}$ \\
\hline $\mathrm{AlFe}_{3} 0.2 \mathrm{Ge}$ & $\begin{array}{l}-0.675 \\
-0.62\end{array}$ & $\begin{array}{l}0.31 \\
0.31\end{array}$ & $\begin{array}{l}\text { N/A } \\
\text { N/A }\end{array}$ & $\begin{array}{l}0 \mathrm{M} \mathrm{KCl} \text { in aerated boric-borate solution ( } \mathrm{pH} 8.4) \\
0.1 \mathrm{M} \mathrm{KCl} \text { in aerated boric-borate solution ( } \mathrm{pH} 8.4)\end{array}$ & $\begin{array}{l}{[67]} \\
{[67]}\end{array}$ \\
\hline $\mathrm{Al}_{3} \mathrm{Fe}$ & $\begin{array}{l}-0.493 \\
-0.539 \\
-0.566 \\
-0.834 \\
-0.510 \\
-0.539 \\
-0.408 \\
-0.23\end{array}$ & $\begin{array}{c}0.442 \\
0.106 \\
-0.382 \\
\mathrm{~N} / \mathrm{A} \\
-0.050 \\
0.106 \\
0.04 \\
0.55\end{array}$ & $\begin{array}{l}\text { N/A } \\
2.1 \\
\text { N/A } \\
\text { N/A } \\
-42^{\mathrm{c}} \\
-160^{\mathrm{c}} \\
-120^{\mathrm{c}} \\
-110^{\mathrm{c}}\end{array}$ & $\begin{array}{c}\text { Aerated } 0.01 \mathrm{M} \mathrm{NaCl} \\
\text { Aerated } 0.1 \mathrm{M} \mathrm{NaCl} \\
\text { Aerated } 0.6 \mathrm{M} \mathrm{NaCl} \\
\text { De-aerated } 1.0 \mathrm{M} \mathrm{AlCl} \\
0.1 \mathrm{M} \mathrm{NaCl}, \mathrm{pH} 2.5 \\
0.1 \mathrm{M} \mathrm{NaCl}, \mathrm{pH} 6 \\
0.1 \mathrm{M} \mathrm{NaCl}, \mathrm{pH} 10 \\
0.1 \mathrm{M} \mathrm{NaCl}, \mathrm{pH} 12.5\end{array}$ & $\begin{array}{l}{[11]} \\
{[11]} \\
{[11]} \\
{[34]} \\
{[34]} \\
{[34]} \\
{[34]}\end{array}$ \\
\hline $\mathrm{MgZn}_{2}(\eta$ phase $)$ & $\begin{array}{l}-1.004 \\
-1.13 \\
-1.001 \\
-1.029 \\
-1.095 \\
-1.015 \\
-1.007 \\
-1.003 \\
-0.999 \\
-1.012\end{array}$ & $\begin{array}{l}\text { No } \\
\text { No } \\
\text { No } \\
\text { No } \\
\text { No } \\
\text { No } \\
\text { N/A } \\
\text { N/A } \\
\text { N/A } \\
\text { N/A }\end{array}$ & $\begin{array}{c}167 \\
4.471 \\
\mathrm{~N} / \mathrm{A} \\
84 \\
\mathrm{~N} / \mathrm{A} \\
30.7 \\
120^{\mathrm{c}} \\
1000^{\mathrm{c}} \\
500^{\mathrm{c}} \\
480^{\mathrm{c}}\end{array}$ & $\begin{array}{c}3.5 \mathrm{wt} . \% \mathrm{NaCl} \\
3.5 \mathrm{wt} . \% \mathrm{NaCl} \\
\text { Aerated } 0.01 \mathrm{M} \mathrm{NaCl} \\
\text { Aerated } 0.1 \mathrm{M} \mathrm{NaCl} \\
\text { Aerated } 0.6 \mathrm{M} \mathrm{NaCl} \\
\text { Neutral } 3.5 \% \mathrm{NaCl} \\
0.1 \mathrm{M} \mathrm{NaCl}, \mathrm{pH} 2.5 \\
0.1 \mathrm{M} \mathrm{NaCl}, \mathrm{pH} 6 \\
0.1 \mathrm{M} \mathrm{NaCl}, \mathrm{pH} 10 \\
0.1 \mathrm{M} \mathrm{NaCl}, \mathrm{pH} 10\end{array}$ & $\begin{array}{l}{[69]} \\
{[70]} \\
{[11]} \\
{[11]} \\
{[11]} \\
{[71]} \\
{[34]} \\
{[34]} \\
{[34]} \\
{[34]}\end{array}$ \\
\hline
\end{tabular}


Table 1. Cont.

\begin{tabular}{|c|c|c|c|c|c|}
\hline Stoichiometry & $E_{\text {corr }}, \mathrm{V}_{\mathrm{SCE}}$ & $E_{\mathrm{pit}}, \mathrm{V}_{\mathrm{SCE}}$ & $i_{\text {corr }} \mu \mathrm{A} / \mathrm{cm}^{2}$ & Environment & Reference \\
\hline $\mathrm{Mg}_{2} \mathrm{Zn}_{11}$ & -1.13 & No & 1.608 & 3.5 wt. $\% \mathrm{NaCl}$ & [70] \\
\hline \multirow{15}{*}{$\mathrm{Al}_{20} \mathrm{Cu}_{2} \mathrm{Mn}_{3}$} & -0.68 & -0.44 & 1.7 & $0.5 \mathrm{M} \mathrm{NaCl}, \mathrm{pH} 9.17$ & [48] \\
\hline & -0.98 & -0.22 & 7.9 & $0.5 \mathrm{M} \mathrm{NaCl}+10 \mathrm{mM} \mathrm{NaVO}_{3}, \mathrm{pH} 9.17$ & [48] \\
\hline & -0.550 & -0.210 & $\mathrm{~N} / \mathrm{A}$ & Aerated $0.01 \mathrm{M} \mathrm{NaCl}$ & [11] \\
\hline & -0.565 & -0.428 & 0.34 & Aerated $0.1 \mathrm{M} \mathrm{NaCl}$ & [11] \\
\hline & -0.617 & -0.534 & N/A & Aerated $0.6 \mathrm{M} \mathrm{NaCl}$ & [11] \\
\hline & -0.742 & -0.33 & 0.22 & Aerated $0.1 \mathrm{M} \mathrm{NaCl}, 10^{\circ} \mathrm{C}$ & [33] \\
\hline & -0.669 & -0.366 & 0.55 & Aerated $0.1 \mathrm{M} \mathrm{NaCl}, 30^{\circ} \mathrm{C}$ & [33] \\
\hline & -0.792 & -0.423 & 1.04 & Aerated $0.1 \mathrm{M} \mathrm{NaCl}, 50^{\circ} \mathrm{C}$ & [33] \\
\hline & -0.871 & -0.385 & 1.34 & Aerated $0.1 \mathrm{M} \mathrm{NaCl}, 70^{\circ} \mathrm{C}$ & [33] \\
\hline & -0.636 & -0.292 & 0.17 & $0.1 \mathrm{M} \mathrm{NaCl}+4 \mathrm{mM} \mathrm{CeCl}_{3}, 30^{\circ} \mathrm{C}$ & {$[51]$} \\
\hline & -0.452 & -0.242 & 0.08 & $0.1 \mathrm{M} \mathrm{NaCl}+4 \mathrm{mM} \mathrm{LaCl}_{3}, 30^{\circ} \mathrm{C}$ & [42] \\
\hline & -0.519 & -0.246 & 0.072 & $0.1 \mathrm{M} \mathrm{NaCl}+4 \mathrm{mM} \mathrm{PrCl}_{3}, 30^{\circ} \mathrm{C}$ & [42] \\
\hline & -0.79 & -0.208 & 0.022 & $0.1 \mathrm{M} \mathrm{NaCl}+1 \mathrm{mM} \mathrm{K}_{2} \mathrm{CrO}_{4}, 30^{\circ} \mathrm{C}$ & [42] \\
\hline & -0.924 & -0.408 & 0.57 & $0.1 \mathrm{M} \mathrm{NaCl}+4 \mathrm{mM} \mathrm{CeCl}_{3}, 50^{\circ} \mathrm{C}$ & [51] \\
\hline & -0.968 & -0.465 & 0.42 & $0.1 \mathrm{M} \mathrm{NaCl}+4 \mathrm{mM} \mathrm{CeCl}_{3}, 70^{\circ} \mathrm{C}$ & [51] \\
\hline \multirow{3}{*}{$\mathrm{Au}_{20} \mathrm{Cu}_{2}(\mathrm{FeMn})_{3}$} & $-0.334^{\mathrm{a}}$ & $\mathrm{N} / \mathrm{A}$ & $\mathrm{N} / \mathrm{A}$ & $0.1 \mathrm{M} \mathrm{Na}_{2} \mathrm{SO}_{4}+0.005 \mathrm{M} \mathrm{NaCl}, \mathrm{pH} 6$ & [31] \\
\hline & $-0.386^{\mathrm{a}}$ & N/A & N/A & $0.1 \mathrm{M} \mathrm{Na}_{2} \mathrm{SO}_{4}+0.005 \mathrm{M} \mathrm{NaCl}, \mathrm{pH} 3$ & [31] \\
\hline & $-0.327^{\mathrm{a}}$ & N/A & $\mathrm{N} / \mathrm{A}$ & $0.1 \mathrm{M} \mathrm{Na}_{2} \mathrm{SO}_{4}+0.005 \mathrm{M} \mathrm{NaCl}, \mathrm{pH} 10$ & [31] \\
\hline \multirow{19}{*}{$\mathrm{Al}_{7} \mathrm{Cu}_{2} \mathrm{Fe}$} & -0.535 & -0.451 & $-320^{c}$ & $0.1 \mathrm{M} \mathrm{NaCl}, \mathrm{pH} 2.5$ & [34] \\
\hline & -0.551 & -0.448 & $-420^{c}$ & $0.1 \mathrm{M} \mathrm{NaCl}, \mathrm{pH} 6$ & [34] \\
\hline & -0.604 & -0.42 & $-2400^{c}$ & $0.1 \mathrm{M} \mathrm{NaCl}, \mathrm{pH} 10$ & [34] \\
\hline & -0.594 & -0.41 & $-2600^{c}$ & $0.1 \mathrm{M} \mathrm{NaCl}, \mathrm{pH} 12.5$ & [34] \\
\hline & -0.63 & -0.38 & 5.8 & $0.5 \mathrm{M} \mathrm{NaCl}, \mathrm{pH} 9.17$ & [48] \\
\hline & -0.93 & -0.14 & 1.2 & $0.5 \mathrm{M} \mathrm{NaCl}+10 \mathrm{mM} \mathrm{NaVO}_{3}, \mathrm{pH} 9.17$ & [48] \\
\hline & -0.549 & -0.447 & N/A & Aerated $0.01 \mathrm{M} \mathrm{NaCl}$ & [11] \\
\hline & -0.551 & -0.448 & 6.3 & Aerated $0.1 \mathrm{M} \mathrm{NaCl}$ & [11] \\
\hline & -0.654 & -0.580 & $\mathrm{~N} / \mathrm{A}$ & Aerated $0.6 \mathrm{M} \mathrm{NaCl}$ & [11] \\
\hline & -0.795 & -0.397 & 0.27 & Aerated $0.1 \mathrm{M} \mathrm{NaCl}, 10^{\circ} \mathrm{C}$ & [33] \\
\hline & -0.695 & -0.454 & 0.66 & Aerated $0.1 \mathrm{M} \mathrm{NaCl}, 30^{\circ} \mathrm{C}$ & [33] \\
\hline & -0.787 & -0.430 & 1.04 & Aerated $0.1 \mathrm{M} \mathrm{NaCl}, 50^{\circ} \mathrm{C}$ & [33] \\
\hline & -0.785 & -0.448 & 2.67 & Aerated $0.1 \mathrm{M} \mathrm{NaCl}, 70^{\circ} \mathrm{C}$ & [33] \\
\hline & -0.602 & -0.301 & 0.15 & $0.1 \mathrm{M} \mathrm{NaCl}+4 \mathrm{mM} \mathrm{CeCl}_{3}, 30^{\circ} \mathrm{C}$ & [51] \\
\hline & -0.661 & -0.29 & 0.18 & $0.1 \mathrm{M} \mathrm{NaCl}+4 \mathrm{mM} \mathrm{LaCl}_{3}, 30^{\circ} \mathrm{C}$ & [42] \\
\hline & -0.731 & -0.371 & 0.97 & $0.1 \mathrm{M} \mathrm{NaCl}+4 \mathrm{mM} \mathrm{PrCl}_{3}, 30^{\circ} \mathrm{C}$ & [42] \\
\hline & -0.874 & -0.101 & 0.05 & $0.1 \mathrm{M} \mathrm{NaCl}+1 \mathrm{mM} \mathrm{K}_{2} \mathrm{CrO}_{4}, 30^{\circ} \mathrm{C}$ & [42] \\
\hline & -0.916 & -0.456 & 1.4 & $0.1 \mathrm{M} \mathrm{NaCl}+4 \mathrm{mM} \mathrm{CeCl}_{3}, 50^{\circ} \mathrm{C}$ & [51] \\
\hline & -1.09 & -0.465 & 1.7 & $0.1 \mathrm{M} \mathrm{NaCl}+4 \mathrm{mM} \mathrm{CeCl}_{3}, 70^{\circ} \mathrm{C}$ & [51] \\
\hline
\end{tabular}

a data is read from the figures in the reference; ${ }^{\mathrm{b}} \mathrm{pH}$ varies from 3 to 11 ; $^{\mathrm{c}}$ current density at a potential of $-0.9 \mathrm{~V}_{\mathrm{SCE}}$ N/A, data is not reported in the literature; No, not applicable. 


\section{Electrochemical Properties of Al-Rich Solid Solutions}

\subsection{Al-Cu Solid Solution}

$\mathrm{Cu}$ is the main alloying element in 2xxx aluminum alloys. It increases mechanical strength through solid solution strengthening and precipitate hardening. The maximum solubility of $\mathrm{Cu}$ in $\mathrm{Al}$ is about $5 \mathrm{wt} . \%$ at $548{ }^{\circ} \mathrm{C}$, but its solubility dramatically decreases as temperature decreases, enabling $2 \mathrm{xxx}$ aluminum alloys to be heat-treatable alloys. $\mathrm{Cu}$ shows complex effects on the corrosion resistance of $\mathrm{Al}$ alloys, depending on its existing form in the alloy [1,2].

Dissolved $\mathrm{Cu}$ in an $\mathrm{Al}$ matrix provides an increase in corrosion resistance [72]. Thermodynamically, pure $\mathrm{Al}$ is classified as very active with a corrosion potential around $-1.2 \mathrm{~V}_{\mathrm{SCE}}$ in $0.1 \mathrm{M} \mathrm{NaCl}$ [13]. However, in neutral aqueous solutions, an oxide film protects it. The corrosion potential of $\mathrm{Al}$ in $0.1 \mathrm{M} \mathrm{NaCl}$ solution increases from -1.2 to $-0.9 \mathrm{~V}_{\mathrm{SCE}}$ by the addition of $4 \mathrm{wt}$. \% $\mathrm{Cu}$ [13]. Addition of $5.2 \mathrm{wt}$. \% Cu also increases the pitting potential, by about $200 \mathrm{mV}$. Kim et al. also reported an elevated pitting potential achieved by the addition of $\mathrm{Cu}$ in $\mathrm{Al}$ and attributed this to inhibition of metastable pitting [72]. Cu enrichment occurring on the surface during potentiostatic and cyclic polarization (without damaging the passive film) enhances the noble behavior [73]. Plots of the pitting potential of $\mathrm{Al}-4 \mathrm{Cu}$ and $\mathrm{Al}-0.2 \mathrm{Cu}$ solid solutions vs. $\mathrm{NaCl}$ concentration $(0.01 \mathrm{M} \sim 5 \mathrm{M})$ under de-aerated conditions can be described by the following equations, respectively [74]:

$$
\begin{gathered}
E(\mathrm{Al}-4 \mathrm{Cu})=-0.881-0.046 \log [\mathrm{NaCl}] \mathrm{V}_{\mathrm{SCE}} \\
E(\mathrm{Al}-0.2 \mathrm{Cu})=-0.101-0.073 \log [\mathrm{NaCl}] \mathrm{V}_{\mathrm{SCE}}
\end{gathered}
$$

The pitting potential of $\mathrm{Al}-\mathrm{Cu}$ solid solution shifted from -0.759 to $-0.648 \mathrm{~V}_{\mathrm{SCE}}$ as the concentration of $\mathrm{Cu}$ increased from $0.011 \mathrm{wt}$. \% to $8.15 \mathrm{wt}$. \% in $1 \mathrm{M} \mathrm{NaCl}$ solution (pH 10) [53]. In this work, the microstructure also included secondary phase $\mathrm{Al}_{2} \mathrm{Cu}$.

However, Ramgopal and Frankel proposed that the addition of $\mathrm{Cu}$ has an effect on dissolution kinetics [75]. They found that the exchange current density slightly increased with the addition of $\mathrm{Cu}$, and the Tafel slope increased even more. This resulted in a higher surface overpotential, shifting the pitting potential to more positive values. It was found that the $\mathrm{Cu}$ did not affect the first stage of repassivation of metastable pitting but tended to extend the second stage as a result of a slower mass transportation rate. This phenomenon was attributed to $\mathrm{Cu}$ accumulation on the surface.

$\mathrm{Cu}$ combines with $\mathrm{Al}$ and other elements to form secondary particle during solidification and heat treatment, and the induced heterogeneous structure causes the alloy to be susceptible to localized corrosion. For example, when $\theta^{\prime \prime}$ precipitation predominates in an $\mathrm{Al}-\mathrm{Cu}$ alloy, aging for a certain time decreases the pitting potential [76]. Upon application of $0.1 \%$ proof stress without aging, the corrosion potential of $\mathrm{Al}-\mathrm{Cu}$ in $1 \mathrm{M} \mathrm{NaCl}+1$ wt. $\% \mathrm{H}_{2} \mathrm{O}_{2}$ exhibited very little change $\left(-0.583\right.$ to $\left.-0.589 \mathrm{~V}_{\mathrm{SCE}}\right)$ [52]. The potential drop is attributed to the decrease in the concentration of dissolved $\mathrm{Cu}$ [53]. Furthermore, $\mathrm{Cu}$ depletion at the grain boundary also increases the pitting susceptibility. The pitting potential of $\mathrm{Al}-4 \% \mathrm{Cu}$ at grain boundaries and within grains is dependent on the chloride concentration ( $\mathrm{pH}=10)$, and the relationship can be described by Equations (3) and (4), respectively [52]:

$$
\begin{gathered}
E(\text { grain boundary })=-0.98-0.085 \log \left[\mathrm{Cl}^{-}\right] \mathrm{V}_{\mathrm{SCE}} \\
E(\text { with grain })=-0.86-0.065 \log \left[\mathrm{Cl}^{-}\right] \mathrm{V}_{\mathrm{SCE}}
\end{gathered}
$$

In addition to a decrease in pitting resistance, the galvanic interaction between $\mathrm{Al}_{2} \mathrm{Cu}$ and adjacent matrix can also lead to localized trenching corrosion. $\mathrm{Al}_{2} \mathrm{Cu}$ possesses a higher corrosion potential than the matrix, and acts as a cathode in localized galvanic corrosion, supporting oxygen reduction. These reactions increase the local $\mathrm{pH}$ near $\mathrm{Al}_{2} \mathrm{Cu}$, induce selective dissolution of $\mathrm{Al}$ in the adjacent matrix, and cause the formation of $\mathrm{Cu}$ nanoparticles [77]. Then, the $\mathrm{Cu}$-rich matrix becomes noble relative to the remote matrix. This leads to galvanic corrosion of matrix areas that are remote from 
the original particles [78]. Hence, the presence of $\mathrm{Cu}$ in IMC particles is detrimental to corrosion resistance, while $\mathrm{Cu}$ dissolved in the matrix is beneficial. However, compared to other elements such as $\mathrm{Fe}, \mathrm{Mn}, \mathrm{Mg}$ and $\mathrm{Si}, \mathrm{Cu}$ is less harmful with regard to pit formation and pitting dissolution (in $5.25 \% \mathrm{NaCl}+2.98 \% \mathrm{H}_{2} \mathrm{O}_{2}$ solution) [53].

\subsection{Al-Mn Solid Solution}

Mn is the main alloying element in 3xxx alloys, but it is also added in 7xxx alloys to improve the ultimate yield strength and fatigue toughness without harming ductility [79]. The addition of Mn in $\mathrm{Al}$ does not adversely affect the corrosion properties. This is generally attributed to the similar electrochemical properties of $\mathrm{Al}_{6} \mathrm{Mn}$ precipitates to $\mathrm{Al}$. A $4.94 \mathrm{wt}$. $\% \mathrm{Mn}$ addition, which exists primarily in the form of secondary particles, slightly increases the pitting potential of $\mathrm{Al}$ by around $40 \mathrm{mV}$ in $1 \mathrm{M}$ $\mathrm{NaCl}$ solution ( $\mathrm{pH}$ 10) [53]. Furthermore, dissolved $\mathrm{Mn}(2 \mathrm{wt} . \%)$ also shifts the corrosion potential positively by $132 \mathrm{mV}$ in $53 \mathrm{~g} / \mathrm{L} \mathrm{NaCl}+3 \mathrm{~g} / \mathrm{L} \mathrm{H}_{2} \mathrm{O}_{2}$ [54], which is consistent with data reported by Reding and Newport [80]. It was also reported that the detrimental effect on corrosion resistance arising from Fe impurities can be alleviated by increasing the Mn concentration. This is attributed to increased $\mathrm{Mn}$ in Fe-containing particles, which suppresses oxygen reduction reactions on these cathodic particles [81].

\subsection{Al-Si Solid Solution}

$\mathrm{Si}$ is the main alloying element in $4 \mathrm{xxx}$ and 6xxx alloys. It can reduce melting temperature and improve fluidity. Although Si possesses a higher corrosion potential than the matrix by a few hundred $\mathrm{mV}$, its effect on the corrosion properties of these alloys is negligible due to its low ability to support cathodic reactions even at a high level of polarization [82,83]. For example, a $1.66 \mathrm{wt}$ \% Si addition in Al-Si binary alloys only slightly increases the pitting potential by around $60 \mathrm{mV}$ in $1 \mathrm{M} \mathrm{NaCl}$ solution at $\mathrm{pH} 10$ [53].

\subsection{Al-Mg Solid Solution}

$\mathrm{Mg}$ is the main alloying element in 5xxx aluminum alloys and it can increase the strength through solid solution strengthening and precipitate strengthening, without detrimental effects on corrosion resistance. Slightly negligible effects on pitting potential are observed when its concentration is high. The pitting potential of Al- $\mathrm{Mg}$ solid solution (less than $0.51 \mathrm{wt} . \% \mathrm{Mg}$ ) in $1 \mathrm{M} \mathrm{NaCl}$ solution (pH 10) is around $-0.76 \mathrm{~V}_{\mathrm{SCE}}$, but it is decreased by $60 \mathrm{mV}$ as the $\mathrm{Mg}$ concentration is increased to $4.51 \mathrm{wt}$. \%. The $\mathrm{Mg}$ concentration in commercial alloys ranges from $0.5 \mathrm{wt}$. \% to $6 \mathrm{wt}$. \%. Inappropriate heat treatment can lead to the formation of a continuous network of $\mathrm{Mg}_{2} \mathrm{Al}_{3}$ at grain boundaries and $\mathrm{Mg}$ depletion, making the alloys susceptible to stress corrosion cracking $[84,85]$.

\subsection{Al-Zn Solid Solution}

$\mathrm{Zn}$ is the main alloying element in 7xxx Al alloys with typical concentrations of about 5-6 wt. \%. $\mathrm{Zn}$ may also be present in other alloys as an acceptable impurity element. The addition of Zn into $\mathrm{Al}$ decreases the corrosion potential. For example, a decrease of more than $200 \mathrm{mV}$ was found with 4 wt. $\% \mathrm{Zn}$ addition in a $53 \mathrm{~g} / \mathrm{L} \mathrm{NaCl}+3 \mathrm{~g} / \mathrm{L} \mathrm{H}_{2} \mathrm{O}_{2}$ solution [54]. Furthermore, the corrosion rate of $\mathrm{Al}-\mathrm{Zn}$ alloys also increases with increasing $\mathrm{Zn}$ concentration. For example, in a $144 \mathrm{~h}$ free corrosion test in $1 \mathrm{M} \mathrm{H}_{2} \mathrm{SO}_{4}$, the corrosion rate is increased by more than 7 times as $\mathrm{Zn}$ concentration increases from $1 \mathrm{wt}$. \% to $2 \mathrm{wt}$. \% [86]. This high increase in corrosion kinetics elevates the susceptibility to stress corrosion cracking [87]. However, some studies show that $\mathrm{Zn}$ improves resistance to stress corrosion cracking in $\mathrm{Al}-\mathrm{Mg}$ alloys by increasing the heterogeneity and eliminating the continuity of $\mathrm{Mg}_{2} \mathrm{Al}_{3}$ precipitates at the grain boundary [88]. 


\section{Electrochemical Properties of Secondary Phase Particles}

\section{1. $\mathrm{Mg} / \mathrm{Li}$-Containing Particles}

$\mathrm{Mg}_{2} \mathrm{Si}-$

$\mathrm{Mg}_{2} \mathrm{Si}$ is a common precipitate in $6 \times x \times \mathrm{Al}$ alloys. $\mathrm{Mg}_{2} \mathrm{Si}$ possesses a lower corrosion potential than the matrix and, therefore, corrodes as an anode during localized galvanic corrosion with the cathodic matrix. Notably, though, the selective dissolution of Mg leads to Si enrichment on the surface, revealing an incongruent dissolution morphology, thereby reversing the particle to a cathode and resulting in the subsequent anodic dissolution of surrounding matrix $[60,61]$. However, owing to the low catalytic ability of $\mathrm{Si}$ to support cathodic reactions, the induced corrosion is expected to be low $[82,83]$. $\mathrm{Mg}_{2} \mathrm{Si}$ preferentially precipitates at grain boundaries by heterogeneous nucleation processes, but these precipitates are less harmful with regards to intergranular corrosion cracking than particles containing other noble alloying elements such as $\mathrm{Cu}$ [82].

$\mathrm{Mg}_{2} \mathrm{Al}_{3}-$

$\mathrm{Mg}_{2} \mathrm{Al}_{3}$ precipitates are commonly found in $5 \mathrm{xxx} \mathrm{Al}$ alloys. Their formation at grain boundaries can lead to intergranular corrosion cracking, referred to as sensitization. This sensitization arises from the active electrochemical properties of $\mathrm{Mg}_{2} \mathrm{Al}_{3}$, which is active relative to the matrix. $\mathrm{Mg}_{2} \mathrm{Al}_{3}$ also passivates but with a relatively large current density $\left(0.1 \mathrm{~mA} / \mathrm{cm}^{2}\right)$. The pitting potential of these precipitates is generally lower than the corrosion potential of $5 x x x$ alloys. This leads to a significant dissolution of $\mathrm{Mg}_{2} \mathrm{Al}_{3}$ during alloy exposure in aqueous solutions, and results in the formation of pits. These corrosion behaviors are detrimental to stress corrosion cracking resistance [89], especially if continuous $\mathrm{Mg}_{2} \mathrm{Al}_{3}$ particles form at grain boundaries due to inappropriate heat treatment or service conditions. Furthermore, $\mathrm{Mg}$ depletion at grain boundaries because of $\mathrm{Mg}_{2} \mathrm{Al}_{3}$ precipitates also degrades the corrosion resistance of these alloys.

\section{$\mathrm{Al}_{2} \mathrm{CuMg}-$}

$\mathrm{Al}_{2} \mathrm{CuMg}$ is a spherical particle formed during solidification or aging and is very active, and only $\mathrm{Al}_{2} \mathrm{CuMg}$ corrodes when AA2024-T3 alloy is exposed to $0.5 \mathrm{M} \mathrm{NaCl}$ for $30 \mathrm{~min}$ [24]. The open circuit potential of $\mathrm{Al}_{2} \mathrm{CuMg}$ in $0.5 \mathrm{M} \mathrm{NaCl}$ is about $-0.93 \mathrm{~V}_{\mathrm{SCE}}$, and it is independent of the solution aeration [21]. The low potential indicates $\mathrm{Al}_{2} \mathrm{CuMg}$ is more active than the matrix, and corrodes. However, the corrosion morphology shows corrosion also occurs on the adjacent matrix [12]. It is suggested that $\mathrm{Al}_{2} \mathrm{CuMg}$ initially dissolve as an anode by the selective dissolution of $\mathrm{Mg}$ and $\mathrm{Al}$, leaving a Cu-rich layer with a porous structure. The dealloying kinetics is accelerated at high temperature in $\mathrm{NaCl}$ solution, as revealed by fluctuations observed during open circuit measurements [33]. $\mathrm{Cu}$ enrichment on the IMC surface leads to an increase in its corrosion potential, which can be higher than the open circuit value of the matrix, thus reversing the galvanic relationship between $\mathrm{Al}_{2} \mathrm{CuMg}$ and the matrix. However, the remnants are frequently not stable because of high local surface area and can also be physically released from the substrate. Although the potential value at unreleased particles is much lower than typical reversible potential values for $\mathrm{Cu}$ redox processes, released particles can be oxidized by dissolved oxygen in water [22]. The curvature of these particles can have a strong effect on their electrochemical properties. The effect on the corrosion potential is described by the following equation $[22,77]$ :

$$
E_{C u}^{\prime \prime}=E_{C u}-\frac{2 \gamma_{C u} \Omega_{C u}}{n F r}
$$

where $\gamma_{\mathrm{Cu}}$ is surface energy $\left(\mathrm{J} / \mathrm{cm}^{2}\right), \Omega_{\mathrm{Cu}}$ is the volume of per molar $\mathrm{Cu}\left(7.1 \mathrm{~cm}^{3} / \mathrm{mol}\right), r$ is the radius of curvature $(\mathrm{cm}), E_{\mathrm{Cu}}$ is the equilibrium potential of $\mathrm{Cu}(\mathrm{V}), n$ is the charge of the ion number of equivalents of charge per mole of reactant, $F$ is Faraday's constant (96,500 C/equiv). 
The redistribution behavior of the enriched $\mathrm{Cu}$ particles is of interest. One possibility is detachment from the original site. Solution movement can enhance detachment and additionally result in relocation of the particle as it is caught up in the movement of the surrounding voluminous corrosion products. After oxidation by dissolved $\mathrm{O}_{2}$ in the solution, $\mathrm{Cu}$ ions can be reduced and deposited on the surrounding matrix, which is also detected on Al-Cu-Mn-Fe particles [90]. These two kinds of $\mathrm{Cu}$, detached $\mathrm{Cu}$ clusters and redeposited $\mathrm{Cu}$, trigger secondary pitting in the matrix [22].

$\mathrm{Cu}$-rich remnants are good cathodic sites for the oxygen reduction reaction, even more efficient than pure $\mathrm{Cu}, \mathrm{Al}$ and $\mathrm{Mg}$ [21]. No individual or combination of those three elements supported the measured high oxygen reduction reaction on the $\mathrm{Cu}$-rich remnants observed in $\mathrm{NaCl}$ solutions. Buchheit attributes this to the remnants' spongy structure and large surface area arising from the selective corrosion of $\mathrm{Al}$ and $\mathrm{Mg}$ [21]. Scully suggests the enhanced reaction is due to $\mathrm{Cu}$ clusters in the $\mathrm{Al}$ oxide film, which improves the conductivity of the $\mathrm{Al}$ oxide film [32].

\section{$\mathrm{Al}_{2} \mathrm{CuLi}-$}

Similar to $\mathrm{Al}_{2} \mathrm{CuMg}, \mathrm{Al}_{2} \mathrm{CuLi}$ is an active particle due to the presence of $\mathrm{Li}$, and has a low corrosion potential of $-1.096 \mathrm{~V}_{\mathrm{SCE}}$ in $\mathrm{NaCl}$ solution. This value decreases further with increasing $\mathrm{pH}$. $\mathrm{Al}_{2} \mathrm{CuLi}$ acts as an anode relative to the matrix. Its corrosion rate is lowered by a factor of about 25 as $\mathrm{pH}$ increases from 3 to 11 [62]. Its corrosion behavior is similar to that of $\mathrm{Al}_{2} \mathrm{CuMg}$ : it initially corrodes as an anode through selective dissolution of $\mathrm{Li}$, and then switches to cathodic behavior, inducing corrosion of the surrounding matrix $[23,62,63,71]$.

$\mathrm{MgZn}_{2}-$

$\mathrm{MgZn}_{2}$ is a common precipitate in $7 \mathrm{xxx}$ alloys. Its corrosion potential is about $-1 \mathrm{~V}_{\mathrm{SCE}}$, which is much lower than the corrosion potential of $7 \mathrm{xxx}$ alloys. Therefore, $\mathrm{MgZ \textrm {Z } _ { 2 }}$ corrodes as an anode relative to the matrix, and $\mathrm{Mg}$ selectively dissolves in the early stages and migrates to the surface, forming a thick magnesium hydroxycarbonate film which passivates the surface $[10,91,92]$. Metallic $\mathrm{Zn}$ mixed with $\mathrm{Mg}$ oxides are found under this film. Unlike the dealloying of $\mathrm{Al}_{2} \mathrm{CuMg}$, the corroded $\mathrm{MgZn}_{2}$ remains active due to $\mathrm{Zn}$ enrichment on the surface. However, the alloy corrosion kinetics decreases with the formation of the film.

\subsection{Cu-Containing Particles}

\section{$\mathrm{Al}_{2} \mathrm{Cu}-$}

$\mathrm{Al}_{2} \mathrm{Cu}$ is a fine and hardening phase formed during aging or heat treatment with a fraction of about 5.4\% in the particle population in AA2014-T3 [93]. Recent work shows they also demonstrate a spherical shape, making them indistinguishable from the $\mathrm{Al}_{2} \mathrm{CuMg}$ [8]. Its corrosion potential is $-0.565 \mathrm{~V}_{\mathrm{SCE}}$ in aerated $0.1 \mathrm{M} \mathrm{NaCl}$ solution [11]. This value is slightly more positive in $0.005 \mathrm{M} \mathrm{NaCl}+0.1 \mathrm{M} \mathrm{Na}_{2} \mathrm{SO}_{4}$ solution (about $-0.4 \mathrm{~V}_{\mathrm{SCE}}$ ) [32]. Still, its corrosion potential is higher than that of the matrix and it is expected to play a cathodic role in localized corrosion processes. $\mathrm{Al}_{2} \mathrm{Cu}$ is an efficient cathodic site which supports the oxygen reduction reaction due to the presence of $\mathrm{Cu}[74,94]$, and the reaction kinetics is independent of $\mathrm{pH}[29,34,39]$. However, the absence of Fe and $\mathrm{Mn}$ (which also facilitate the oxygen reduction reaction) results in $\mathrm{Al}_{2} \mathrm{Cu}$ having a lower reaction efficiency than $\mathrm{Al}_{7} \mathrm{Cu}_{2} \mathrm{Fe}$ and $\mathrm{Al}_{20} \mathrm{Cu}_{2} \mathrm{Mn}_{3}$ [95].

The pitting potential of $\mathrm{Al}_{2} \mathrm{Cu}$ as a function of $\mathrm{Cl}^{-}$concentration in de-aerated $\mathrm{NaCl}$ solution can be described by the following equation [74]:

$$
E=-0.885-0.055 \log [\mathrm{NaCl}] \mathrm{V}_{\mathrm{SCE}}
$$

$\mathrm{Al}_{2} \mathrm{Cu}$ corrodes by selective dissolution of $\mathrm{Al}$ during exposure to neutral $\mathrm{NaCl}$. $\mathrm{Cu}$ may also dissolve during anodic polarization, but the generated oxidized $\mathrm{Cu}$ can be reduced when the applied 
potential is lower than the equilibrium potential of metallic $\mathrm{Cu}$ [57]. These $\mathrm{Cu}$ ions can deposit on the bulk sample, and induce corrosion on the surrounding bare matrix. It is suggested that $\mathrm{Cu}$ redistribution and enrichment arising from this phenomenon are dominated by $\mathrm{Al}_{2} \mathrm{Cu}$ (and $\left.\mathrm{Al}_{2} \mathrm{CuMg}\right)$ dissolution during the initial stages of exposure [96]. However, Vukmirovic reported that deposited $\mathrm{Cu}$ ions are from $\mathrm{Al}_{2} \mathrm{CuMg}$, not from $\mathrm{Al}_{2} \mathrm{Cu}$ [97]. The selective dissolution of $\mathrm{Al}$ was also explored to fabricate $\mathrm{Cu}$ catalysts with high surface areas. For example, porous $\mathrm{Cu}\left(\mathrm{Raney}^{\mathrm{TM}} \mathrm{Cu}\right)$ with a high surface area can be obtained by immersing $\mathrm{Al}_{2} \mathrm{Cu}$ in $6 \mathrm{M} \mathrm{NaOH}$ at $274 \mathrm{~K}$ [98].

$\mathrm{Al}_{7} \mathrm{Cu}_{2} \mathrm{Fe}-$

$\mathrm{Al}_{7} \mathrm{Cu}_{2} \mathrm{Fe}$ is one kind of irregular constituent compound and comprises about $6.8 \%$ of particles by number [93]. The mean equivalent diameter of these particles is $1.7 \mu \mathrm{m}$ and the number density is $220 / \mathrm{mm}^{2}$. During cold working, these particles often break and align into bands parallel to the rolling direction. $\mathrm{Al}_{7} \mathrm{Cu}_{2} \mathrm{Fe}$ has a corrosion potential of about $-550 \mathrm{mV}_{\mathrm{SCE}}$ in $0.1 \mathrm{M} \mathrm{NaCl}$, which decreases with increasing chloride concentration [11]. Its noble corrosion potential relative to the matrix demonstrates its cathodic role in localized corrosion. Furthermore, it supports oxygen reduction at a rate higher than $\mathrm{Al}_{2} \mathrm{Cu}$ and $\mathrm{Al}_{20} \mathrm{Cu}_{2} \mathrm{Mn}_{3}$ [39]. This behavior is attributed to the presence of $\mathrm{Fe}$, which is an effective cathodic site for the oxygen reduction reaction [99]. It was also found that its ability to support oxygen reduction increases with increasing $\mathrm{pH}$ [39]. Circumferential pitting commonly occurs at the matrix adjacent to this particle, resulting in a 'trenching' morphology. These localized pits are possible stress concentration sites and can initiate cracks [100].

$\mathrm{Al}_{20} \mathrm{Cu}_{2} \mathrm{Mn}_{3}-$

$\mathrm{Al}_{20} \mathrm{Cu}_{2} \mathrm{Mn}_{3}$ is a common, fine rod-shaped dispersoid with a homogeneous distribution in the matrix. The corrosion potential of $\mathrm{Al}_{20} \mathrm{Cu}_{2} \mathrm{Mn}_{3}$ in $0.1 \mathrm{M} \mathrm{Na}_{2} \mathrm{SO}_{4}+0.1 \mathrm{M} \mathrm{NaCl}$ is about $-0.5 \mathrm{~V}_{\mathrm{SCE}}$ [29], and it was slightly lower $\left(-0.565 \mathrm{~V}_{\mathrm{SCE}}\right)$ in $0.1 \mathrm{M} \mathrm{NaCl}$ alone [11]. Like $\mathrm{Al}_{7} \mathrm{Cu}_{2} \mathrm{Fe}$, it also acts as a cathode because of its relatively more positive corrosion potential relative to the surrounding matrix. Furthermore, it shows enhanced oxygen reduction reaction kinetics when compared to pure aluminum. This is attributed to $\mathrm{Cu}$ clusters present in the Al oxide film on the particle surface, which increases the conduction of electrons through the oxide film [29]. Ilevbare and Scully reported the impedance of $\mathrm{Al}_{20} \mathrm{Cu}_{2}(\mathrm{MnFe})_{3}$ in $\mathrm{NaCl}$ and $\mathrm{Na}_{2} \mathrm{SO}_{4}$ solution is high, indicating that these compounds are resistant to self-dissolution, while $\mathrm{Al}_{2} \mathrm{CuMg}$ has a relatively lower impedance and is prone to active dissolution [32]. This was also observed by Leblanc and Frankel using atomic force microscopy (AFM) [24], which showed no localized corrosion at Al-Cu-Mn-Fe particles but significant attack at $\mathrm{Al}_{2} \mathrm{CuMg}$ after exposure to $0.5 \mathrm{M} \mathrm{NaCl}$ solution. These particles were identified using Scanning Kelvin probe force microscopy, and related to the corrosion morphology obtained using AFM.

\section{3. $\mathrm{Fe} / \mathrm{Ti} / \mathrm{Zr} / \mathrm{Ta}$-Containing Particles}

\section{$\mathrm{Al}_{3} \mathrm{Fe}-$}

Iron is a main impurity element in $1 \times x x \mathrm{Al}$ alloys and it generally combines with $\mathrm{Al}$ to form $\mathrm{Al}_{3} \mathrm{Fe}$ due to its extremely low solubility in $\mathrm{Al}[1,4]$. Fe accelerates pit formation compared to other binary alloying elements $\left(\mathrm{Mg}, \mathrm{Mn}, \mathrm{Si}, \mathrm{Cu}\right.$ ), when the concentration is higher than $0.6 \mathrm{wt}$. \% [53]. $\mathrm{Al}_{3} \mathrm{Fe}$ induces corrosion of the surrounding matrix acting as a cathode due to its high corrosion potential relative to the Al-rich matrix and is, therefore, detrimental to the corrosion resistance of these alloys [11]. The effect of this galvanic corrosion is attributed to its good catalytic ability to support oxygen reduction reactions and hydrogen evolution [101]. However, incorporation of $\mathrm{Mn}$ in Fe-containing particles can alleviate galvanic interactions by decreasing the potential difference between the particles and the matrix [81]. A study by Rosalbino et al. shows that the addition of $\mathrm{Si}$ and $\mathrm{Ge}$ in $\mathrm{Al}-\mathrm{Fe}$ particles $\left(\mathrm{AlFe}_{3}\right)$ improves passivation properties and increases pitting resistance [67]. 
$\mathrm{Al}_{3} \mathrm{Ta} / \mathrm{Al}_{3} \mathrm{Zr} / \mathrm{Al}_{3} \mathrm{Ti}-$

Owing to its higher corrosion potential, $\mathrm{Al}_{3} \mathrm{Ta}$ acts as a cathode relative to the surrounding $\mathrm{Al}$-solid solution matrix, thereby inducing dissolution of the surrounding matrix [59]. The presence of Ta makes the electrochemical properties of $\mathrm{Al}_{3} \mathrm{Ta}$ much different than those dissolved in pure $\mathrm{Al}$, improving the catalytic ability to support the oxygen reduction reaction and hydrogen evolution reactions. This is attributed to a diminished oxide film and enrichment of $\mathrm{Ta}$ (conductive $\mathrm{Ta}_{2} \mathrm{O}_{5}$ ) on the surface [102]. However, the cathodic kinetics on $\mathrm{Al}_{3} \mathrm{Ta}$ and $\mathrm{Al}_{3} \mathrm{Zr}$ particles are much slower than those on $\mathrm{Al}_{2} \mathrm{Cu}$ and $\mathrm{Al}_{7} \mathrm{Cu}_{2} \mathrm{Fe}[11,39,59,102]$, and the induced corrosion of adjacent matrix is negligible. Ta also suppresses the passive current density due to the formation of stable $\mathrm{Ta}_{2} \mathrm{O}_{5}$ at $\mathrm{pH}$ values less than 12 . The electrochemical behaviors of $\mathrm{Al}_{3} \mathrm{Zr}$ and $\mathrm{Al}_{3} \mathrm{Ti}$ are similar to $\mathrm{Al}_{3} \mathrm{Ta}$ [102]. It is also notable that only very small amounts of $\mathrm{Zr}$, Ta and Ti are found in these alloys and the particle size that includes these elements is nano-scale. Their effects on the corrosion resistance of alloys are not substantial.

\section{Research Outlook on Localized Galvanic Corrosion Arising between an Al-Rich Matrix and Particles}

Galvanic corrosion is a crucial issue in the localized corrosion of aluminum alloys, which arises from the heterogeneous microstructure of these alloys. Pitting as a result of galvanic reactions can be initiation sites for cracks, leading to a failure. Extensive studies have been conducted to investigate the electrochemical properties of secondary phase particles under different conditions using the microcell method [11,17,34,42]. Electrochemical characterization can be employed to elucidate the mechanism of localized corrosion, analyze the driving force of corrosion and investigate the oxygen reduction reaction kinetics on cathodic sites. However, the evolution of surface conditions, as galvanic corrosion proceeds, leads to significant changes in the corrosion properties of cathodic and anodic regions and the associated corrosion kinetics. The corrosion process cannot be fully demonstrated from corrosion measurements on a single compound. Therefore, a direct galvanic corrosion study is necessary to provide supplementary corrosion information for better interpreting localized corrosion. Furthermore, galvanic corrosion data can also provide experimental validation for galvanic corrosion simulation.

Most work about galvanic corrosion is conducted using dissimilar metals which are connected via mechanical 'bonding' or through wires $[103,104]$. The mechanically 'bonded' samples are obtained through rolling or other mechanical methods. However, questions arise about the possibility of a gap between the two 'bonded' metal blocks described in such work. Any gap between the metal blocks that allows water molecules to penetrate will induce crevice corrosion. The importance of understanding interfacial energy at the interface of secondary phase particles and the alloy matrix makes wire-bonded samples inappropriate for studying galvanic corrosion in alloys.

Diffusion couples, which are fabricated using two dissimilar metals subjected to a heat process, can be employed to study galvanic corrosion between secondary phase particles and the alloy matrix. Diffusion couples can consist of IMCs adjacent to a solid solution matrix [105-107] and can serve as a model of the heterogeneous microstructure of an alloy. The size of a fabricated matrix with IMCs is typically a few hundred micrometers depending on the heat treatment time and temperature. The diffusion-controlled formation eliminates the gap between IMCs and the matrix, which excludes the influence of crevice corrosion. Electrochemical tests and monitoring of corrosion morphology evolution can then be performed using these fabricated couples. This method provides an easy way to simulate the heterogeneous structure of metallic alloys and study the electrochemical interactions between particles and the alloy matrix. Diffusion couples also avoid the problem of crevice corrosion associated with other methods and they provide comprehensive and accurate corrosion data for galvanic corrosion studies. This electrochemical characterization data can be used to analyze corrosion mechanisms and provide guidance on corrosion mitigation. It can also illustrate the corrosion behavior of heterogeneous structures and can guide alloy development. Additionally, interactions between inhibitors and galvanic couples provide a direct evaluation of inhibition performance on 
localized galvanic corrosion, and can be used to quickly screen inhibitors for corrosion mitigation. Furthermore, the corrosion morphology examined in this type of work can serve as experimental validation for galvanic corrosion modeling.

\section{Summary}

The heterogeneous structure of $\mathrm{Al}$ alloys can increase their mechanical strength but also render them susceptible to the localized corrosion due to the different electrochemical properties between an Al-rich matrix and secondary phase particles. The galvanic interactions rely on their electrochemical properties, which are strongly dependent on the surrounding environment, and are also key to understand pit formation. The electrochemical characteristics collected on synthesized alloy ingots or on single phase samples, using electrochemical microcell methods, are summarized and reviewed in this work. However, as mentioned above, the corrosion behavior of some particles such as $\mathrm{Al}_{2} \mathrm{CuMg}$ can be complex, and care should be taken because experimental parameters can also affect the result. Therefore, the data reported in the literature may not be applicable under certain environmental conditions and should be used with caution. Although extensive studies have been undertaken to characterize corrosion properties, as shown in Table 1, presenting the data in a form that is complete and useable can be very challenging [108], and will require further work.

Acknowledgments: Thanks are given to the financial supports from the National Natural Science Foundation of China (51604046) and the Fundamental Research Funds for the Central Universities (106112016CDJXY130005).

Conflicts of Interest: The authors declare no conflict of interest.

\section{References}

1. Hatch, J.E. Aluminum: Properties and Physical Metallurgy; American Society for Metals: Metals Park, OH, USA, 1984.

2. Polmear, I.J. Light Alloys: Metallurgy of the Light Metals, 3rd ed.; J. Wiley \& Sons: Metals Park, OH, USA, 1981.

3. Vargel, C. Corrosion of Aluminium, 1st ed.; Elsevier: Amsterdam, The Netherlands; Boston, MA, USA, 2004.

4. Mondolfo, L.F. Aluminum Alloys: Structure and Properties; Butterworths: London, UK, 1976.

5. Davis, J. Corrosion: Understanding the Basics; ASM International: Materials Park, OH, USA, 2000.

6. Davis, J.R. Corrosion of Aluminum and Aluminum Alloys; ASM International: Materials Park, OH, USA, 1999.

7. Buchheit, R.G. A compilation of corrosion potentials reported for intermetallic phases in aluminum-alloys. J. Electrochem. Soc. 1995, 142, 3994-3996. [CrossRef]

8. Boag, A.; Hughes, A.E.; Wilson, N.C.; Torpy, A.; MacRae, C.M.; Glenn, A.M.; Musterb, T.H. How complex is the microstructure of AA2024-T3? Corros. Sci. 2009, 51, 1565-1568. [CrossRef]

9. Revie, R.W.; Uhlig, H.H. Corrosion and Corrosion Control: An Introduction to Corrosion Science and Engineering, 4th ed.; Wiley-Interscience: Hoboken, NJ, USA, 2008.

10. Frayne, C. Shreir's Corrosion; Elsevier Science \& Technology: London, UK, 2010.

11. Birbilis, N.; Buchheit, R.G. Electrochemical characteristics of intermetallic phases in aluminum alloys-An experimental survey and discussion. J. Electrochem. Soc. 2005, 152, B140-B151. [CrossRef]

12. Buchheit, R.G.; Grant, R.P.; Hlava, P.F; Mckenzie, B.; Zender, G.L. Local dissolution phenomena associated with $\mathrm{S}$ phase $\left(\mathrm{Al}_{2} \mathrm{CuMg}\right)$ particles in aluminum alloy 2024-T3. J. Electrochem. Soc. 1997, 144, 2621-2628. [CrossRef]

13. Baek, Y.; Frankel, G.S. Electrochemical quartz crystal microbalance study of corrosion of phases in AA2024. J. Electrochem. Soc. 2003, 150, B1-B9. [CrossRef]

14. Wang, S.C.; Starink, M.J. Two types of S phase precipitates in Al-Cu-Mg alloys. Acta Mater. 2007, 55, 933-941. [CrossRef]

15. Yoon, Y.; Buchheit, R.G. Dissolution behavior of $\mathrm{Al}_{2} \mathrm{CuMg}$ (S phase) in chloride and chromate conversion coating solutions. J. Electrochem. Soc. 2006, 153, B151-B155. [CrossRef]

16. Xu, D.K.; Birbilis, N.; Rometsch, P.A. Effect of S-phase dissolution on the corrosion and stress corrosion cracking of an As-rolled Al-Zn-Mg-Cu alloy. Corrosion 2012, 68. [CrossRef] 
17. Cavanaugh, M.K.K.; Li, J.C.; Birbilis, N.; Buchheit, R.G.G. Electrochemical characterization of intermetallic phases common to aluminum alloys as a function of solution temperature. J. Electrochem. Soc. 2014, 161, C535-C543. [CrossRef]

18. Yasakau, K.A.; Zheludkevich, M.L.; Lamaka, S.V.; Ferreira, M.G.S. Role of intermetallic phases in localized corrosion of AA5083. Electrochim. Acta 2007, 52, 7651-7659. [CrossRef]

19. Scholes, F.H.; Hughes, A.E.; Jamieson, D.; Inoue, K.; Furman, S.A.; Muster, T.H.; Hardin, S.G.; Lau, D.; Harvey, T.G.; Corrigan, P.; et al. Interaction of $\mathrm{Ce}(\mathrm{dbp})_{3}$ with surface of aluminium alloy 2024-T3 using macroscopic models of intermetallic phases. Corros. Eng. Sci. Technol. 2009, 44, 416-424. [CrossRef]

20. Dimitrov, N.; Mann, J.A.; Vukmirovic, M.; Sieradzki, K. Dealloying of $\mathrm{Al}_{2} \mathrm{CuMg}$ in alkaline media. J. Electrochem. Soc. 2000, 147, 3283-3285. [CrossRef]

21. Buchheit, R.G.; Montes, L.P.; Martinez, M.A.; Michael, J.; Hlava, P.F. The electrochemical characteristics of bulk-synthesized $\mathrm{Al}_{2}$ CuMg. J. Electrochem. Soc. 1999, 146, 4424-4428. [CrossRef]

22. Buchheit, R.G.; Martinez, M.A.; Montes, L.P. Evidence for $\mathrm{Cu}$ ion formation by dissolution and dealloying the $\mathrm{Al}_{2} \mathrm{CuMg}$ intermetallic compound in rotating ring-disk collection experiments. J. Electrochem. Soc. 2000, 147, 119-124. [CrossRef]

23. Buchheit, R.G. The Electrochemistry of $\theta\left(\mathrm{Al}_{2} \mathrm{Cu}\right), \mathrm{S}\left(\mathrm{Al}_{2} \mathrm{CuMg}\right)$ and $\mathrm{T}\left(\mathrm{Al}_{2} \mathrm{CuLi}\right)$ and localized corrosion and environment assisted cracking in high strength Al alloys. Mater. Sci. Forum 2000, 331-337, 1641-1646. [CrossRef]

24. Leblanc, P.; Frankel, G.S. A study of corrosion and pitting initiation of AA2024-T3 using atomic force microscopy. J. Electrochem. Soc. 2002, 149, B239-B247. [CrossRef]

25. Guillaumin, V.; Mankowski, G. Localized corrosion of 2024 T351 aluminium alloy in chloride media. Corros. Sci. 1999, 41, 421-438. [CrossRef]

26. DeRose, J.A.; Suter, T.; Balkowiec, A.; Michalski, J.; Kurzydlowski, K.J.; Schmutz, P. Localised corrosion initiation and microstructural characterisation of an $\mathrm{Al} 2024$ alloy with a higher $\mathrm{Cu}$ to $\mathrm{Mg}$ ratio. Corros. Sci. 2012, 55, 313-325. [CrossRef]

27. Birbilis, N.; Cavanaugh, M.K.; Kovarik, L.; Buchheit, R.G. Nano-scale dissolution phenomena in Al-Cu-Mg alloys. Electrochem. Commun. 2008, 10, 32-37. [CrossRef]

28. Schneider, O.; Ilevbare, G.O.; Kelly, R.G.; Scully, J.R. In situ confocal laser scanning microscopy of AA2024-T3 corrosion metrology. III. Underfilm corrosion of epoxy-coated AA2024-T3. J. Electrochem. Soc. 2007, 154, C397-C410. [CrossRef]

29. Ilevbare, G.O.; Scully, J.R. Oxygen reduction reaction kinetics on chromate conversion coated Al-Cu, $\mathrm{Al}-\mathrm{Cu}-\mathrm{Mg}$, and Al-Cu-Mn-Fe intermetallic compounds. J. Electrochem. Soc. 2001, 148, B196-B207. [CrossRef]

30. Schneider, O.; Ilevbare, G.O.; Scully, J.R.; Kelly, R.G. In situ confocal laser scanning microscopy of AA2024-T3 corrosion metrology. II. Trench formation around particles. J. Electrochem. Soc. 2004, 151, B465-B472. [CrossRef]

31. Ilevbare, G.O.; Schneider, O.; Kelly, R.G.; Scully, J.R. In situ confocal laser scanning microscopy of AA 2024-T3 corrosion metrology. I. Localized corrosion of particles. J. Electrochem. Soc. 2004, 151, B453-B464. [CrossRef]

32. Ilevbare, G.O.; Scully, J.R. Mass-Transport-Limited oxygen reduction reaction on AA2024-T3 and selected intermetallic compounds in chromate-containing solutions. Corrosion 2001, 57, 134-152. [CrossRef]

33. Li, J.; Hurley, B.; Buchheit, R. Effect of temperature on the localized corrosion of aa2024-t3 and the electrochemistry of intermetallic compounds during exposure to a dilute $\mathrm{NaCl}$ solution. Corrosion 2016, 72, 1281-1291. [CrossRef]

34. Birbilis, N.; Buchheit, R.G. Investigation and discussion of characteristics for intermetallic phases common to aluminum alloys as a function of solution pH. J. Electrochem. Soc. 2008, 155, C117-C126. [CrossRef]

35. Bohni, H.; Suter, T.; Schreyer, A. Microtechniques and nanotechniques to study localized corrosion. Electrochim. Acta 1995, 40, 1361-1368. [CrossRef]

36. Suter, T.; Muller, Y.; Schmutz, P.; von Trzebiatowski, O. Microelectrochemical studies of pit initiation on high purity and ultra high purity aluminum. Adv. Eng. Mater. 2005, 7, 339-348. [CrossRef]

37. Suter, T.; Webb, E.G.; Bohni, H.; Alkire, R.C. Pit initiation on stainless steels in $1 \mathrm{M} \mathrm{NaCl}$ with and without mechanical stress. J. Electrochem. Soc. 2001, 148, B174-B185. [CrossRef]

38. Suter, T.; Bohni, H. Microelectrodes for studies of localized corrosion processes. Electrochim. Acta 1998, 43, 2843-2849. [CrossRef] 
39. Birbilis, N.; Cavanaugh, M.K.; Buchheit, R.G. Electrochemical behavior and localized corrosion associated with $\mathrm{Al}_{7} \mathrm{Cu}_{2} \mathrm{Fe}$ particles in aluminum alloy 7075-T651. Corros. Sci. 2006, 48, 4202-4215. [CrossRef]

40. Birbilis, N.; Padgett, B.N.; Buchheit, R.G. Limitations in microelectrochemical capillary cell testing and transformation of electrochemical transients for acquisition of microcell impedance data. Electrochim. Acta 2005, 50, 3536-3544. [CrossRef]

41. Li, J.; Sun, W.; Hurley, B.; Luo, A.A.; Buchheit, R.G. Cu redistribution study during the corrosion of AZ91 using a rotating ring-disk collection experiment. Corros. Sci. 2016, 112, 760-764. [CrossRef]

42. Li, J.; Hurley, B.; Buchheit, R. Microelectrochemical characterization of the effect of rare earth inhibitors on the localized corrosion of AA2024-T3. J. Electrochem. Soc. 2015, 162, C563-C571. [CrossRef]

43. Li, J.; Birbilis, N.; Buchheit, R.G. Electrochemical assessment of interfacial characteristics of intermetallic phases present in aluminium alloy 2024-T3. Corros. Sci. 2015, 101, 155-164. [CrossRef]

44. Rajan, V.; Neelakantan, L. Communication-Local electrochemical study using droplet cell microscopy on a rough surface. J. Electrochem. Soc. 2016, 163, C704-C706. [CrossRef]

45. Rajan, V.; Neelakantan, L. On the corrosion behavior of $\mathrm{Al}_{2} \mathrm{Cu}$ by local electrochemical impedance spectroscopy using droplet cell microscopy. J. Solid State Electrochem. 2017, 21, 603-609. [CrossRef]

46. Li, J.; Buchheit, R. Development of zinc ferrocyanide ion exchange compounds for corrosion-inhibiting and sensing pigments. Prog. Org. Coat. 2016, 104, 210-216. [CrossRef]

47. Li, J.; Hurley, B.; Buchheit, R. Inhibition performance study of vanadate on AA2024-T3 at high temperature by SEM, FIB, Raman and XPS. J. Electrochem. Soc. 2015, 162, C219-C227. [CrossRef]

48. Ralston, K.D.; Young, T.L.; Buchheit, R.G. Electrochemical evaluation of constituent intermetallics in aluminum alloy 2024-T3 exposed to aqueous vanadate inhibitors. J. Electrochem. Soc. 2009, 156, C135-C146. [CrossRef]

49. Li, J. Electrochemical Characterization of Intermetallic Compounds in AA2024-T3 and Localized Corrosion Morphology of Al-Cu-Mg at Elevated Temperature; The Ohio State University: Columbus, OH, USA, 2015.

50. Afseth, A.; Nordlien, J.H.; Scamans, G.M.; Nisancioglu, K. Filiform corrosion of binary aluminium model alloys. Corros. Sci. 2002, 44, 2529-2542. [CrossRef]

51. Li, J.; Belinda, H.; Buchheit, R. Inhibition performance study of cerium inhibitors on the localized corrosion of AA2024-T3 as a function of temperature. J. Electrochem. Soc. 2016, 163, C845-C852. [CrossRef]

52. Sugimoto, K.; Hoshino, K.; Kageyama, M.; Kageyama, S.; Sawada, Y. Stress corrosion cracking of aged Al4\%Cu alloy in $\mathrm{NaCl}$ solution. Corros. Sci. 1975, 15, 709-720. [CrossRef]

53. Sugimoto, K.; Sawada, Y.; Morioka, S. Effects of alloying elements on the pitting corrosion of Aluminum. Trans. Jpn. Inst. Met. 1972, 13, 345-351. [CrossRef]

54. Anderson, W.A.; Stumpf, H.C. Technical note: Effects of Manganese on the electrode or free corrosion potentials of Aluminum. Corrosion 1980, 36, 212-213. [CrossRef]

55. Tohma, K. Intergranular corrosion mechanism in Al-Mn alloys. J. Jpn. Inst. Met. Mater. 1982, 46, $973-979$.

56. Niskanen, P.; Sanders, T.H.; Rinker, J.G.; Marek, M. Corrosion of aluminum alloys containing lithium. Corros. Sci. 1982, 22, 283-304. [CrossRef]

57. Mazurkiewicz, B.; Piotrowski, A. The electrochemical behavior of the $\mathrm{Al}_{2} \mathrm{Cu}$ intermetallic compound. Corros. Sci. 1983, 23, 697-707. [CrossRef]

58. Fleming, K.M.; Zhu, A.; Scully, J.R. Corrosion of AA6061 brazed with an Al-Si alloy: Effects of Si on metallurgical and corrosion behavior. Corrosion 2012, 68, 1126-1145. [CrossRef]

59. Buchheit, R.G. The Electrochemical behavior of the $\mathrm{Al}_{3}$ Ta intermetallic compound and pitting in two-phase Al-Ta alloys. J. Electrochem. Soc. 1995, 142, 51-58. [CrossRef]

60. Zeng, F.L.; Wei, Z.L.; Li, J.F.; Li, C.X.; Tan, X.; Zhang, Z.; Zheng, Z.Q. Corrosion mechanism associated with $\mathrm{Mg}_{2} \mathrm{Si}$ and Si particles in Al-Mg-Si alloys. Trans. Nonferr. Met. Soc. China 2011, 21, 2559-2567. [CrossRef]

61. Gupta, R.K.; Sukiman, N.L.; Fleming, K.M.; Gibson, M.A.; Birbilis, N. Electrochemical behavior and localized corrosion associated with $\mathrm{Mg}_{2} \mathrm{Si}$ particles in $\mathrm{Al}$ and $\mathrm{Mg}$ alloys. ECS Electrochem. Lett. 2012, 1, C1-C3. [CrossRef]

62. Buchheit, R.G.; Moran, J.P.; Stoner, G.E. Electrochemical behavior of the $\mathrm{T} 1\left(\mathrm{Al}_{2} \mathrm{CuLi}\right)$ intermetallic compound and its role in localized corrosion of Al-2\%Li-3\%Cu alloys. Corrosion 1994, 50, 120-130. [CrossRef]

63. Li, J.F.; Li, C.X.; Peng, Z.W.; Chen, W.J.; Zheng, Z.Q. Corrosion mechanism associated with T1 and T2 precipitates of Al-Cu-Li alloys in $\mathrm{NaCl}$ solution. J. Alloys Compd. 2008, 460, 688-693. [CrossRef] 
64. Leard, R.R.; Buchheit, R.G. Electrochemical characterization of copper-bearing intermetallic compounds and localized corrosion of Al-Cu-Mg-Mn alloy 2024. Alum. Alloys 2002, 396, 1491-1496. [CrossRef]

65. Lyndon, J.A.; Gupta, R.K.; Gibson, M.A.; Birbilis, N. Electrochemical behaviour of the $\beta$-phase intermetallic $\left(\mathrm{Mg}_{2} \mathrm{Al}_{3}\right)$ as a function of $\mathrm{pH}$ as relevant to corrosion of aluminium-magnesium alloys. Corros. Sci. 2013, 70, 290-293. [CrossRef]

66. Montagné, P.; Tillard, $\mathrm{M} . \mathrm{Mg}_{2} \mathrm{Al}_{3}$, a complex and disordered intermetallic compound as anode material for metal-air batteries. J. Solid State Electrochem. 2015, 19, 685-695. [CrossRef]

67. Rosalbino, F.; Carlini, R.; Parodi, R.; Zanicchi, G.; Scavino, G. Investigation of passivity and its breakdown on $\mathrm{Fe}_{3} \mathrm{Al}-\mathrm{Si}$ and $\mathrm{Fe}_{3} \mathrm{Al}-\mathrm{Ge}$ intermetallics in chloride-containing solution. Corros. Sci. 2014, 85, 394-400. [CrossRef]

68. Seri, O.; Imaizumi, M. The dissolution of $\mathrm{FeAl}_{3}$ intermetallic compound and deposition on aluminum in $\mathrm{AlCl}_{3}$ solution. Corros. Sci. 1990, 30, 1121-1133. [CrossRef]

69. Yao, C.; Zhu, T.; Chen, W.; Tay, S.; Gao, W. Corrosion behaviour of $\mathrm{MgZn}_{2}$ alloy using electrochemical methods. In Proceedings of the 8th Structural Integrity and Fracture, Melbourne, Australia, 11-12 July 2013.

70. Byun, J.M.; Yu, J.M.; Kim, D.K.; Kim, T.-Y.; Jung, W.-S.; Kim, Y.D. Corrosion behavior of $\mathrm{Mg}_{2} \mathrm{Zn}_{11}$ and $\mathrm{MgZn}_{2}$ single phases. J. Korean Inst. Met. Mater. 2013, 51. [CrossRef]

71. Li, J.F.; Zheng, Z.Q.; Li, S.C.; Chen, W.J.; Ren, W.D.; Zhao, X.S. Simulation study on function mechanism of some precipitates in localized corrosion of Al alloys. Corros. Sci. 2007, 49, 2436-2449. [CrossRef]

72. Kim, Y.; Buchheit, R.G. A characterization of the inhibiting effect of $\mathrm{Cu}$ on metastable pitting in dilute $\mathrm{Al}-\mathrm{Cu}$ solid solution alloys. Electrochim. Acta 2007, 52, 2437-2446. [CrossRef]

73. Kim, Y.; Buchheit, R.G.; Kotula, P.G. Effect of alloyed Cu on localized corrosion susceptibility of Al-Cu solid solution alloys-Surface characterization by XPS and STEM. Electrochim. Acta 2010, 55, 7367-7375. [CrossRef]

74. Galvele, J.R.; de De Micheli, S.M. Mechanism of intergranular corrosion of Al-Cu alloys. Corros. Sci. 1970, 10, 795-807. [CrossRef]

75. Ramgopal, T.; Frankel, G.S. Role of alloying additions on the dissolution kinetics of aluminum binary alloys using artificial crevice electrodes. Corrosion 2001, 57, 702-711. [CrossRef]

76. Muller, I.L.; Galvele, J.R. Pitting potential of high purity binary Aluminum alloys-I. Al-Cu alloys. Pitting and intergranular corrosion. Corros. Sci. 1977, 17, 179-189. [CrossRef]

77. Dimitrov, N.; Mann, J.A.; Sieradzki, K. Cu redistribution during corrosion of aluminum alloys. J. Electrochem. Soc. 1999, 146, 98-102. [CrossRef]

78. Scully, J.R.; Peebles, D.E.; Romig, A.D.; Frear, D.R.; Hills, C.R. Metallurgical factors influencing the corrosion of aluminum, Al-Cu, and Al-Si alloy thin films in dilute hydrofluoric solution. Metall. Trans. A 1992, 23, 2641-2655. [CrossRef]

79. Nam, S.W.; Lee, D.H. The effect of Mn on the mechanical behavior of Al alloys. Met. Mater. 2000, 6, 13-16. [CrossRef]

80. Reding, J.T.; Newport, J.J. Aluminum anodes in sea water. Mater. Prot. 1966, 5, 15-18.

81. Zamin, M. The Role of Mn in the Corrosion Behavior of Al-Mn Alloys. Corrosion 1981, 37, 627-632. [CrossRef]

82. Larsen, M.H.; Walmsley, J.C.; Lunder, O.; Nisancioglu, K. Effect of excess silicon and small copper content on intergranular corrosion of 6000-series Aluminum alloys. J. Electrochem. Soc. 2010, 157, C61. [CrossRef]

83. Mizuno, K.; Nylund, A.; Olefjord, I. Surface reactions during pickling of an aluminium-magnesium-silicon alloy in phosphoric acid. Corros. Sci. 2001, 43, 381-396. [CrossRef]

84. Zhang, R.; Knight, S.P.; Holtz, R.L.; Goswami, R.; Davies, C.H.J.; Birbilis, N. A survey of sensitization in 5xxx series Aluminum alloys. Corrosion 2016, 72, 144-159. [CrossRef]

85. Yan, J.; Heckman, N.M.; Velasco, L.; Hodge, A.M. Improve sensitization and corrosion resistance of an Al-Mg alloy by optimization of grain boundaries. Sci. Rep. 2016, 6, 26870. [CrossRef] [PubMed]

86. Ekuma, C.E.; Idenyi, N.E.; Umahi, A.E. The Effects of Zinc Additions on the corrosion susceptibility of Aluminium alloys in various tetraoxosulphate (VI) acid environments. J. Appl. Sci. 2007, 7, 237-241.

87. Davis, J.R. Alloying: Understanding the Basics; ASM International: Materials Park, OH, USA, 2001.

88. Zakharov, M.V.; Rogel'berg, L.N. Effect of zinc on the susceptibility of aluminum-magnesium alloys to stress corrosion. Met. Sci. Heat Treat. 1963, 5, 692-695. [CrossRef]

89. Shreir, L.L.; Jarman, R.A.; Burstein, G.T. Corrosion-Volume 1. Metal/Environment Reactions; Newnes-Butterworth: London, UK, 1994. 
90. Chen, G.S.; Gao, M.; Wei, R.P. Microconstituent-induced pitting corrosion in aluminum alloy 2024-T3. Corrosion 1996, 52, 8-15. [CrossRef]

91. Diler, E.; Lescop, B.; Rioual, S.; Vien, G.N.; Thierry, D.; Rouvellou, B. Initial formation of corrosion products on pure zinc and $\mathrm{MgZn}_{2}$ examinated by XPS. Corros. Sci. 2014, 79, 83-88. [CrossRef]

92. Oh, M.-S.; Kim, S.-H.; Kim, J.-S.; Lee, J.-W.; Shon, J.-H.; Jin, Y.-S. Surface and cut-edge corrosion behavior of $\mathrm{Zn}-\mathrm{Mg}-\mathrm{Al}$ alloy-coated steel sheets as a function of the alloy coating microstructure. Met. Mater. Int. 2016, 22, 26-33. [CrossRef]

93. Hughes, A.E.; MacRae, C.; Wilson, N.; Torpy, A.; Muster, T.H.; Glenn, A.M. Sheet AA2024-T3: A new investigation of microstructure and composition. Surf. Interface Anal. 2010, 42, 334-338. [CrossRef]

94. Ketcham, S.J.; Haynie, F.H. Electrochemical behavior of aluminum alloys susceptible to intergranular corrosion. I. effect of cooling elate on structure and electrochemical behavior in 2024 Aluminum alloy. Corrosion 1963, 19, 242t-246t. [CrossRef]

95. Nisancioglu, K. Electrochemical-behavior of Aluminum-base intermetallics containing iron. J. Electrochem. Soc. 1990, 137, 69-77. [CrossRef]

96. Buchheit, R.G.; Boger, R.K. Cu Redistribution and Surface Enrichment Due to Dissolution of Al-Cu Alloys. In Proceedings of the Localized Corrosion Proceedings Research Top Symposium Corrosion, Houston, TX, USA, 2001.

97. Vukmirovic, M.B.; Dimitrov, N.; Sieradzki, K. Dealloying and corrosion of Al alloy 2024-T3. J. Electrochem. Soc. 2002, 149, B428-B439. [CrossRef]

98. Smith, A.J.; Tran, T.; Wainwright, M.S. Kinetics and mechanism of the preparation of Raney®copper. J. Appl. Electrochem. 1999, 29, 1085-1094. [CrossRef]

99. Seri, O. The Effect of Nacl Concentration on the corrosion behavior of Aluminum containing iron. Corros. Sci. 1994, 36, 1789-1803. [CrossRef]

100. Pao, P.S.; Feng, C.R.; Gill, S.J. Corrosion fatigue crack initiation in aluminum alloys 7075 and 7050 . Corrosion 2000, 56, 1022-1031. [CrossRef]

101. Buchheit, R.G.; Maestas, L.M.; Sorensen, N.R. The electrochemical behavior of the $\mathrm{Al}_{3} \mathrm{Fe}$ intermetallic compound and localized corrosion of impure 1100 Al. In 183rd Electrochemistry Society Meeting; Electrochemical Society: Pennington, NJ, USA; Honolulu, HI, USA, 1993; pp. 187-198.

102. Scully, J.R.; Knight, T.O; Buchheit, R.G.; Peebles, D.E. Electrochemical characteristics of the $\mathrm{Al}_{2} \mathrm{Cu}_{1} \mathrm{Al}_{3} \mathrm{Ta}$ and $\mathrm{Al}_{3} \mathrm{Zr}$ intermetallic phases and their relevancy to the localized corrosion of $\mathrm{Al}$ alloys. Corros. Sci. 1993, 35, 185-195. [CrossRef]

103. Skar, J.I. Corrosion and corrosion prevention of magnesium alloys. Mater. Corros. 1999, 50, 2-6. [CrossRef]

104. Song, G.; Johannesson, B.; Hapugoda, S.; StJohn, D. Galvanic corrosion of magnesium alloy AZ91D in contact with an aluminium alloy, steel and zinc. Corros. Sci. 2004, 46, 955-977. [CrossRef]

105. Kodentsov, A.; Bastin, G.; van Loo, F.J. The diffusion couple technique in phase diagram determination. J. Alloys Compd. 2001, 320, 207-217. [CrossRef]

106. Zhao, J.-C.; Jackson, M.R.; Peluso, L.A.; Brewer, L.N. A diffusion-multiple approach for mapping phase diagrams, hardness, and elastic modulus. JOM 2002, 54, 42-45. [CrossRef]

107. Zhao, J.-C.; Jackson, M.R.; Peluso, L.A.; Brewer, L.N. A diffusion multiple approach for the accelerated design of structural materials. MRS Bull. 2011, 27, 324-329. [CrossRef]

108. Buchheit, R.G.; Birbilis, N. Electrochemical microscopy: An approach for understanding localized corrosion in microstructurally complex metallic alloys. Electrochim. Acta 2010, 55, 7853-7859. [CrossRef]

(C) 2017 by the authors. Licensee MDPI, Basel, Switzerland. This article is an open access article distributed under the terms and conditions of the Creative Commons Attribution (CC BY) license (http://creativecommons.org/licenses/by/4.0/). 